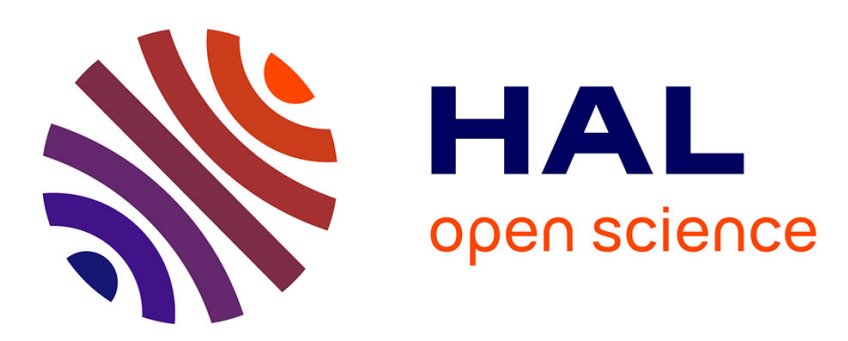

\title{
High-Order Leap-Frog Based Discontinuous Galerkin Method for the Time-Domain Maxwell Equations on Non-Conforming Simplicial Meshes
}

Hassan Fahs

\section{- To cite this version:}

Hassan Fahs. High-Order Leap-Frog Based Discontinuous Galerkin Method for the Time-Domain Maxwell Equations on Non-Conforming Simplicial Meshes. Numerical mathematics: a journal of Chinese universities, 2009, 2 (3), pp.275 - 300. hal-00600467

\section{HAL Id: hal-00600467 https://hal.science/hal-00600467}

Submitted on 10 Jul 2013

HAL is a multi-disciplinary open access archive for the deposit and dissemination of scientific research documents, whether they are published or not. The documents may come from teaching and research institutions in France or abroad, or from public or private research centers.
L'archive ouverte pluridisciplinaire HAL, est destinée au dépôt et à la diffusion de documents scientifiques de niveau recherche, publiés ou non, émanant des établissements d'enseignement et de recherche français ou étrangers, des laboratoires publics ou privés. 


\title{
High-order leap-frog based discontinuous Galerkin method for the time-domain Maxwell equations on non-conforming simplicial meshes
}

\author{
Hassan Fahs* \\ IFP, 1 \& 4 avenue de Bois-Préau, 92852 Rueil-Malmaison Cedex, France.
}

\begin{abstract}
A high-order leap-frog based non-dissipative discontinuous Galerkin timedomain method for solving Maxwell's equations is introduced and analyzed. The proposed method combines a centered approximation for the evaluation of fluxes at the interface between neighboring elements, with a $N$ th-order leap-frog time scheme. Moreover, the interpolation degree is defined at the element level and the mesh is refined locally in a non-conforming way resulting in arbitrary level hanging nodes. The method is proved to be stable under some CFL-like condition on the time step. The convergence of the semi-discrete approximation to Maxwell's equations is established rigorously and bounds on the global divergence error are provided. Numerical experiments with high-order elements show the potential of the method.
\end{abstract}

AMS subject classifications: 65M12, 65M50, 65M60, 74S10, 78A40.

Key words: Maxwell's equations, discontinuous Galerkin method, leap-frog time scheme, stability, convergence, non-conforming meshes, high-order accuracy.

\section{Introduction}

The accurate modeling of systems involving electromagnetic waves, in particular through the resolution of the time-domain Maxwell equations on space grids, remains of strategic interest for many technologies. The still prominent Finite Difference TimeDomain (FDTD) method proposed by Yee [20] lacks two important features to be fully applied in industrial contexts. First, the huge restriction to structured or blockstructured grids. Second, the efficiency of FDTD methods is limited when fully curvilinear coordinates are used. Many different types of methods have been proposed in order to handle complex geometries and heterogeneous media by dealing with unstructured tetrahedral meshes, including, for example, mass lumped Finite Element Time-Domain (FETD) methods [12,14], mimetic methods [11], or Finite Volume TimeDomain (FVTD) methods [17], which all fail in being at the same time efficient, easily extendible to high orders of accuracy, stable, and energy-conserving.

This work was carried out when the author was at INRIA, Nachos team, F-06902 Sophia Antipolis, France. ${ }^{*}$ Corresponding author. Email address: hassan.fahs@gmail.com (H. Fahs) 
Recently, discontinuous Galerkin methods have attracted much research to solve electromagnetic wave propagation problems. Being higher order versions of traditional finite volume methods [13], Discontinuous Galerkin Time-Domain (DGTD) methods based on discontinuous finite element spaces, easily handle elements of various types and shapes, irregular non-conforming meshes $[8,9]$, and even locally varying polynomial degree [8]. They hence offer great flexibility in the mesh design, but also lead to (block-) diagonal mass matrices and therefore yield fully explicit, inherently parallel methods when coupled with explicit time stepping [1]. Moreover, continuity is weakly enforced across mesh interfaces by adding suitable bilinear forms (so-called numerical fluxes) to the standard variational formulations. Whereas high-order DGTD methods have been developed on conforming meshes $[4,5,10]$, the design of non-conforming discontinuous Galerkin time-domain methods is still in its infancy. In practice, the nonconformity can result from a local refinement of the mesh (i.e. h-refinement), of the interpolation degree (i.e. $p$-enrichment) or of both of them (i.e. $h p$-refinement).

This work is concerned with the study of high-order leap-frog schemes that are extensions of the second-order leap-frog scheme adopted in the DGTD methods that are studied in $[8,9]$. The motivation behind this study is to improve the overall accuracy for the same mesh resolution and/or to improve convergence when the mesh resolution is increased. Not surprisingly, the arbitrary high-order DGTD methods discussed in this work are consistently more accurate than the DGTD methods based on the secondorder leap-frog scheme. The high-order leap-frog schemes require more computational operations to update a cell. Fortunately, this can be alleviated by the ability to use discretization meshes with fewer points per wavelength for the same level of accuracy.

This paper is structured as follows. In Sec. 2, we introduce the high-order nonconforming DGTD method for solving the system of Maxwell's equations. Our two main results, the stability and the $h p$-convergence of the proposed method, are stated and proved in Sec. 3. In this section we also establish bounds on the behavior of the divergence error. In Sec. 4 we verify our theoretical results through numerical experiments. Finally, some concluding remarks are presented in Sec. 5.

\section{An arbitrary high-order non-conforming DGTD method}

We consider the Maxwell equations in three space dimensions for heterogeneous anisotropic linear media with no source. The electric permittivity tensor $\overline{\bar{\epsilon}}(x)$ and the magnetic permeability tensor $\overline{\bar{\mu}}(x)$ are varying in space, time-invariant and both symmetric positive definite. The electric field $\overrightarrow{\mathbf{E}}$ and the magnetic field $\overrightarrow{\mathbf{H}}$ verify:

$$
\begin{gathered}
\overline{\bar{\epsilon}} \partial_{t} \overrightarrow{\mathbf{E}}=\operatorname{curl} \overrightarrow{\mathbf{H}}, \quad \overline{\bar{\mu}} \partial_{t} \overrightarrow{\mathbf{H}}=-\operatorname{curl} \overrightarrow{\mathbf{E}}, \\
\operatorname{div}(\overline{\bar{\epsilon}} \overrightarrow{\mathbf{E}})=0, \quad \operatorname{div}(\overline{\bar{\mu}} \overrightarrow{\mathbf{H}})=0,
\end{gathered}
$$

where the symbol $\partial_{t}$ denotes a time derivative. These equations are set and solved on a bounded polyhedral domain $\Omega$ of $\mathbb{R}^{3}$. For the sake of simplicity, a metallic boundary 
condition is set everywhere on the domain boundary $\partial \Omega$, i.e. $\vec{n} \times \overrightarrow{\mathbf{E}}=0$ (where $\vec{n}$ denotes the unitary outwards normal).

\subsection{Space discretization}

We consider a partition $\Omega_{h}$ of $\Omega$ into a set of tetrahedra $\tau_{i}$ of size $h_{i}$ with boundaries $\partial \tau_{i}$ such that $h=\max _{\tau_{i} \in \Omega_{h}} h_{i}$. To each $\tau_{i} \in \Omega_{h}$ we assign an integer $p_{i} \geq 0$ (the local interpolation degree) and we collect the $p_{i}$ in the vector $p=\left\{p_{i}: \tau_{i} \in \Omega_{h}\right\}$. Of course, if $p_{i}$ is uniform in all element $\tau_{i}$ of the mesh, we have $p=p_{i}$. Within this construction we admit meshes with possibly hanging nodes i.e. by allowing nonconforming (or irregular) meshes where element vertices can lie in the interior of faces of other elements. However, we assume that the local mesh sizes and approximation degrees are of bounded variation, that is, there exist a constant $\kappa_{1}>0$, depending only on the shape-regularity of the mesh, and a constant $\kappa_{2}>0$, such that:

$$
\begin{aligned}
& \kappa_{1}^{-1} h_{i} \leq h_{k} \leq \kappa_{1} h_{i} \\
& \kappa_{2}^{-1} p_{i} \leq p_{k} \leq \kappa_{2} p_{i}
\end{aligned}
$$

for all neighboring elements $\tau_{i}$ and $\tau_{k}$ in $\Omega_{h}$. Nevertheless, the above hypothesis is not restrictive in practice and allows, in particular for geometric refinement and linearly increasing approximation degrees. We also assume that $\Omega_{h}$ is shape regular in the sense that there is a constant $\eta>0$ such that:

$$
\forall \tau_{i} \in \Omega_{h}, h_{i} \leq \eta \rho_{i},
$$

where $\rho_{i}$ is the diameter of the insphere of $\tau_{i}$. Each tetrahedron $\tau_{i}$ is assumed to be the image, under a smooth bijective (diffeomorphic) mapping, of a fixed reference tetrahedron $\hat{\tau}=\{\hat{x}, \hat{y}, \hat{z} \mid \hat{x}, \hat{y}, \hat{z} \geq 0 ; \hat{x}+\hat{y}+\hat{z} \leq 1\}$. For each tetrahedron $\tau_{i}$, $\overline{\bar{\epsilon}}_{i}$ and $\overline{\bar{\mu}}_{i}$ are respectively the local electric permittivity and magnetic permeability tensors of the medium, which could be varying inside the element $\tau_{i}$. For two distinct tetrahedra $\tau_{i}$ and $\tau_{k}$ in $\Omega_{h}$, the (non-empty) intersection $\tau_{i} \cap \tau_{k}$ is a convex polyhedron $a_{i k}$ which we will call interface, with unitary normal vector $\vec{n}_{i k}$, oriented from $\tau_{i}$ towards $\tau_{k}$. For the boundary interfaces, the index $k$ corresponds to a fictitious element outside the domain. We denote by $\mathcal{F}_{h}^{I}$ the union of all interior faces of $\Omega_{h}$, by $\mathcal{F}_{h}^{B}$ the union of all boundary faces of $\Omega_{h}$, and by $\mathcal{F}_{h}=\mathcal{F}_{h}^{I} \cup \mathcal{F}_{h}^{B}$. Furthermore, we identify $\mathcal{F}_{h}^{B}$ to $\partial \Omega$ since $\Omega$ is a polyhedron. Finally, we denote by $\mathcal{V}_{i}$ the set of indices of the elements which are neighbors of $\tau_{i}$ (having an interface in common).

In the following, for a given partition $\Omega_{h}$ and vector $p$, we seek approximate solutions to Eq. (2.1) in the finite dimensional subspace $V_{p}\left(\Omega_{h}\right)=\left\{\vec{v} \in L^{2}(\Omega)^{3}: \vec{v}_{\mid \tau_{i}} \in \mathbb{P}_{p_{i}}\left(\tau_{i}\right), \forall \tau_{i} \in\right.$ $\left.\Omega_{h}\right\}$, where $\mathbb{P}_{p_{i}}\left(\tau_{i}\right)$ denotes the space of nodal polynomials of degree at most $p_{i}$ inside the element $\tau_{i}$. Note that the polynomial degree $p_{i}$ may vary from element to element in the mesh. By non-conforming interface we mean an interface $a_{i k}$ which is such that at least one of its vertices is a hanging node, or/and such that $p_{\left.i\right|_{a_{i k}}} \neq p_{\left.k\right|_{a_{i k}}}$. 
Following the discontinuous Galerkin approach, the electric and magnetic fields inside each finite element are seeked for as linear combinations $\left(\overrightarrow{\mathbf{E}}_{i}, \overrightarrow{\mathbf{H}}_{i}\right)$ of linearly independent basis vector fields $\vec{\varphi}_{i j}, 1 \leq j \leq d_{i}$, where $d_{i}$ denotes the local number of degrees of freedom inside $\tau_{i}$. We denote by $\mathcal{P}_{i}=\operatorname{Span}\left(\vec{\varphi}_{i j}, 1 \leq j \leq d_{i}\right)$. The approximate fields $\left(\overrightarrow{\mathbf{E}}_{h}, \overrightarrow{\mathbf{H}}_{h}\right)$, defined by $\left(\forall i, \overrightarrow{\mathbf{E}}_{h \mid \tau_{i}}=\overrightarrow{\mathbf{E}}_{i}, \overrightarrow{\mathbf{H}}_{h \mid \tau_{i}}=\overrightarrow{\mathbf{H}}_{i}\right)$ are allowed to be completely discontinuous across element boundaries. For such a discontinuous field $\overrightarrow{\mathbf{U}}_{h}$, we define its average $\left\{\overrightarrow{\mathbf{U}}_{h}\right\}_{i k}$ through any internal interface $a_{i k}$, as $\left\{\overrightarrow{\mathbf{U}}_{h}\right\}_{i k}=\left(\overrightarrow{\mathbf{U}}_{i \mid a_{i k}}+\overrightarrow{\mathbf{U}}_{k \mid a_{i k}}\right) / 2$. Because of this discontinuity, a global variational formulation cannot be obtained. However, dot-multiplying Eq. (2.1) by any given vector function $\vec{\varphi} \in \mathcal{P}_{i}$, integrating over each single element $\tau_{i}$ and integrating by parts, yields:

$$
\left\{\begin{array}{l}
\int_{\tau_{i}} \vec{\varphi} \cdot \overline{\bar{\epsilon}}_{i} \partial_{t} \overrightarrow{\mathbf{E}}=\int_{\tau_{i}} \operatorname{curl} \vec{\varphi} \cdot \overrightarrow{\mathbf{H}}-\int_{\partial \tau_{i}} \vec{\varphi} \cdot(\overrightarrow{\mathbf{H}} \times \vec{n}) \\
\int_{\tau_{i}} \vec{\varphi} \cdot \overline{\bar{\mu}}_{i} \partial_{t} \overrightarrow{\mathbf{H}}=-\int_{\tau_{i}} \operatorname{curl} \vec{\varphi} \cdot \overrightarrow{\mathbf{E}}+\int_{\partial \tau_{i}} \vec{\varphi} \cdot(\overrightarrow{\mathbf{E}} \times \vec{n}) .
\end{array}\right.
$$

In Eq. (2.5), we now replace the exact fields $\overrightarrow{\mathbf{E}}$ and $\overrightarrow{\mathbf{H}}$ by the approximate fields $\overrightarrow{\mathbf{E}}_{h}$ and $\overrightarrow{\mathbf{H}}_{h}$ in order to evaluate volume integrals. For integrals over $\partial \tau_{i}$, a specific treatment must be introduced since the approximate fields are discontinuous through element faces. We choose to use a fully centered numerical flux, i.e. $\forall i, \forall k \in \mathcal{V}_{i}, \overrightarrow{\mathbf{E}}_{\mid a_{i k}} \simeq$ $\left\{\overrightarrow{\mathbf{E}}_{h}\right\}_{i k}, \overrightarrow{\mathbf{H}}_{\mid a_{i k}} \simeq\left\{\overrightarrow{\mathbf{H}}_{h}\right\}_{i k}$. The metallic boundary condition on a boundary interface $a_{i k}$ (where $k$ is the element index of a fictitious neighboring element) is dealt with weakly, in the sense that traces of fictitious fields $\overrightarrow{\mathbf{E}}_{k}$ and $\overrightarrow{\mathbf{H}}_{k}$ are used for the computation of numerical fluxes for the boundary element $\tau_{i}$. In the present case, where all boundaries are metallic, we simply take $\overrightarrow{\mathbf{E}}_{k \mid a_{i k}}=-\overrightarrow{\mathbf{E}}_{i \mid a_{i k}}$ and $\overrightarrow{\mathbf{H}}_{k \mid a_{i k}}=\overrightarrow{\mathbf{H}}_{i \mid a_{i k}}$. Replacing surface integrals using the centered numerical flux in Eq. (2.5) and re-integrating by parts yields:

$$
\left\{\begin{aligned}
\int_{\tau_{i}} \vec{\varphi} \cdot \overline{\bar{\epsilon}}_{i} \partial_{t} \overrightarrow{\mathbf{E}}_{i} & =\frac{1}{2} \int_{\tau_{i}}\left(\operatorname{curl} \vec{\varphi} \cdot \overrightarrow{\mathbf{H}}_{i}+\operatorname{curl} \overrightarrow{\mathbf{H}}_{i} \cdot \vec{\varphi}\right)-\frac{1}{2} \sum_{k \in \mathcal{V}_{i}} \int_{a_{i k}} \vec{\varphi} \cdot\left(\overrightarrow{\mathbf{H}}_{k} \times \vec{n}_{i k}\right), \\
\int_{\tau_{i}} \vec{\varphi} \cdot \overline{\bar{\mu}}_{i} \partial_{t} \overrightarrow{\mathbf{H}}_{i} & =-\frac{1}{2} \int_{\tau_{i}}\left(\operatorname{curl} \vec{\varphi} \cdot \overrightarrow{\mathbf{E}}_{i}+\operatorname{curl} \overrightarrow{\mathbf{E}}_{i} \cdot \vec{\varphi}\right)+\frac{1}{2} \sum_{k \in \mathcal{V}_{i}} \int_{a_{i k}} \vec{\varphi} \cdot\left(\overrightarrow{\mathbf{E}}_{k} \times \vec{n}_{i k}\right) .
\end{aligned}\right.
$$

We can rewrite this formulation in terms of scalar unknowns. Inside each element, the fields being recomposed according to $\overrightarrow{\mathbf{E}}_{i}=\sum_{1 \leq j \leq d_{i}} E_{i j} \vec{\varphi}_{i j}$ and $\overrightarrow{\mathbf{H}}_{i}=\sum_{1 \leq j \leq d_{i}} H_{i j} \vec{\varphi}_{i j}$. Let us denote by $\mathbf{E}_{i}$ and $\mathbf{H}_{i}$ respectively the column vectors $\left(E_{i l}\right)_{1 \leq l \leq d_{i}}$ and $\left(\bar{H}_{i l}\right)_{1 \leq l \leq d_{i}}$. Eq. (2.6) can be rewritten as:

$$
\left\{\begin{aligned}
M_{i}^{\epsilon} \partial_{t} \mathbf{E}_{i} & =K_{i} \mathbf{H}_{i}-\sum_{k \in \mathcal{V}_{i}} S_{i k} \mathbf{H}_{k} \\
M_{i}^{\mu} \partial_{t} \mathbf{H}_{i} & =-K_{i} \mathbf{E}_{i}+\sum_{k \in \mathcal{V}_{i}} S_{i k} \mathbf{E}_{k}
\end{aligned}\right.
$$


where the symmetric positive definite mass matrices $M_{i}^{\sigma}$ ( $\sigma$ stands for $\epsilon$ or $\mu$ ) and the symmetric stiffness matrix $K_{i}$ (all of size $d_{i} \times d_{i}$ ) are given by :

$$
\begin{aligned}
\left(M_{i}^{\sigma}\right)_{j l} & =\int_{\tau_{i}}{ }^{t} \vec{\varphi}_{i j} \cdot \overline{\bar{\sigma}}_{i} \vec{\varphi}_{i l}, \\
\left(K_{i}\right)_{j l} & =\frac{1}{2} \int_{\tau_{i}}{ }^{t} \vec{\varphi}_{i j} \cdot \operatorname{curl} \vec{\varphi}_{i l}+{ }^{t} \vec{\varphi}_{i l} \cdot \operatorname{curl} \vec{\varphi}_{i j} .
\end{aligned}
$$

For any interface $a_{i k}$, the $d_{i} \times d_{k}$ rectangular matrix $S_{i k}$ is given by:

$$
\left(S_{i k}\right)_{j l}=\frac{1}{2} \int_{a_{i k}}{ }^{t} \vec{\varphi}_{i j} \cdot\left(\vec{\varphi}_{k l} \times \vec{n}_{i k}\right), \quad 1 \leq j \leq d_{i}, 1 \leq l \leq d_{k} .
$$

Note that, if $a_{i k}$ is a conforming interface (i.e. none of its vertices is a hanging node), the matrix $S_{i k}$ is evaluated in a direct way once and for all. However, if $a_{i k}$ is a nonconforming interface, this matrix is strongly dependent on the position of the hanging nodes on the mesh. For that, and only for non-conforming interfaces, we calculate the matrix $S_{i k}$ by using a cubature formula [7].

Finally, if all electric (resp. magnetic) unknowns are gathered in a column vector $\mathbb{E}$ (resp. $\mathbb{H}$ ) of size $d=\sum_{i} d_{i}$, then the space discretized system, Eq. (2.7), can be rewritten as:

$$
\left\{\begin{array}{l}
\mathbb{M}^{\epsilon} \partial_{t} \mathbb{E}=\mathbb{K} \mathbb{H}-\mathbb{A} \mathbb{H}-\mathbb{B H} \\
\mathbb{M}^{\mu} \partial_{t} \mathbb{H}=-\mathbb{K} \mathbb{E}+\mathbb{A E}-\mathbb{B E}
\end{array}\right.
$$

where we have the following definitions and properties:

- $\mathbb{M}^{\epsilon}, \mathbb{M}^{\mu}$ and $\mathbb{K}$ are $d \times d$ block diagonal matrices with diagonal blocks equal to $M_{i}^{\epsilon}, M_{i}^{\mu}$ and $K_{i}$ respectively. Therefore $\mathbb{M}^{\epsilon}$ and $\mathbb{M}^{\mu}$ are symmetric positive definite matrices, and $\mathbb{K}$ is a symmetric matrix.

- $\mathbb{A}$ is also a $d \times d$ block sparse matrix, whose non-zero blocks are equal to $S_{i k}$ when $a_{i k} \in \mathcal{F}_{h}^{I}$. Since $\vec{n}_{k i}=-\vec{n}_{i k}$, it can be checked from Eq. (2.8) that $\left(S_{i k}\right)_{j l}=\left(S_{k i}\right)_{l j}$ and then $S_{k i}={ }^{t} S_{i k}$; thus $\mathbb{A}$ is a symmetric matrix.

- $\mathbb{B}$ is a $d \times d$ block diagonal matrix, whose non-zero blocks are equal to $S_{i k}$ when $a_{i k} \in \mathcal{F}_{h}^{B}$. In that case, $\left(S_{i k}\right)_{j l}=-\left(S_{i k}\right)_{l j}$; thus $\mathbb{B}$ is a skew-symmetric matrix.

One finally obtains that the Maxwell equations, discretized using discontinuous Galerkin finite-elements with centered fluxes and arbitrary local accuracy and basis functions can be written, in function of the matrix $\mathbb{S}=\mathbb{K}-\mathbb{A}-\mathbb{B}$, in the general form:

$$
\left\{\begin{array}{l}
\mathbb{M}^{\epsilon} \partial_{t} \mathbb{E}=\mathbb{S H} \\
\mathbb{M}^{\mu} \partial_{t} \mathbb{H}=-{ }^{t} \mathbb{S E}
\end{array}\right.
$$




\subsection{Time discretization}

In almost all high-order DG formulations, the time-integrator is usually chosen to be some variant of Runge-Kutta (RK). The low storage RK schemes introduced in [6] are among the most popular choices for time integration of the DG space-discretized Maxwell equations. High-order RKDG schemes have been used by Monk and Richter [16] for solving linear symmetric hyperbolic problems, Hesthaven and Warburton [13], Chen et al. [3] and Lu et al. [15] for solving time-domain electromagnetics. A dispersion and dissipation study for a high-order DG method for solving Maxwell's equations have been conducted in [18] using several high-order RK schemes.

In an attempt to offer an alternative to Runge-Kutta schemes, we shall use family of high-order explicit leap-frog (LF) schemes originally proposed by Young [21]. The chief attributes of these integrators are that the memory requirements are small and the algorithmic complexity is not significantly increased, with respect to the second-order leap-frog scheme. We can introduce the $N$ th-order explicit leap-frog $\left(L_{N}\right)$ integrator as an approximation of the solution of the first-order ODE:

$$
\dot{y}(t)=A y(t) \Rightarrow y(t)=e^{A\left(t-t_{0}\right)} y\left(t_{0}\right),
$$

with $y\left(t_{0}\right)$ as initial value and $A$ is a square matrix. The time discrete equivalent of Eq. (2.11) is given by:

$$
y(n \Delta t)=e^{A \Delta t} y((n-1) \Delta t) .
$$

The system of Eq. (2.10) can be rewritten as:

$$
\partial_{t}\left(\begin{array}{c}
\mathbb{E} \\
\mathbb{H}
\end{array}\right)=\underbrace{\left(\begin{array}{cc}
0 & \mathbb{M}^{-\epsilon} \mathbb{S} \\
-\mathbb{M}^{-\mu t_{\mathbb{S}}} & 0
\end{array}\right)}_{\mathcal{A}} \underbrace{\left(\begin{array}{c}
\mathbb{E} \\
\mathbb{H}
\end{array}\right)}_{\mathbb{Y}(t)}
$$

Note that the system matrix $\mathcal{A}$ depends only on the spatial configuration. Seeking a time discrete solution of Eq. (2.13), a discretization in time with a global time step $\Delta t$ is introduced. The time discrete solution of the first-order system of ODEs, Eq. (2.13), is a discretized version of the exponential solution according to its scalar equivalent given by Eq. (2.12):

$$
\mathbb{Y}(n \Delta t)=\Phi(\Delta t) \mathbb{Y}((n-1) \Delta t)
$$

with:

$$
\Phi(\Delta t)=\sum_{i=0}^{\infty} \frac{\Delta t^{i}}{i !} \mathcal{A}^{i}:=e^{\mathcal{A} \Delta t} .
$$

Finally, the solution of Eq. (2.13) is written as: 


$$
\left(\begin{array}{c}
\mathbb{E}(n \Delta t) \\
\mathbb{H}(n \Delta t)
\end{array}\right)=\underbrace{\left(\begin{array}{ll}
\Phi_{11} & \Phi_{12} \\
\Phi_{21} & \Phi_{22}
\end{array}\right)}_{\Phi} \underbrace{\left(\begin{array}{c}
\mathbb{E}((n-1) \Delta t) \\
\mathbb{H}((n-1) \Delta t)
\end{array}\right)}_{\mathbb{Y}((n-1) \Delta t)} .
$$

The time discrete solution, Eqs. (2.14) and (2.16), is exact, as long as $\Phi(\Delta t)$ follows Eq. (2.15). The construction of $N$ th-order integration schemes is based on a truncation of Eq. (2.15) at the $N$ th element, leading to an approximated solution.

In the sequel, superscripts refer to time stations and $\Delta t$ is the global time step. The unknowns related to the electric field are approximated at integer time-stations $t^{n}=n \Delta t$ and are denoted by $\mathbb{E}^{n}$. The unknowns related to the magnetic field are approximated at half-integer time-stations $t^{n+1 / 2}=(n+1 / 2) \Delta t$ and are denoted by $\mathbb{H}^{n+\frac{1}{2}}$. The $N$ th-order explicit leap-frog time integrator can be written in the following way:

$$
\left(\begin{array}{c}
\mathbb{E}^{n+1} \\
\mathbb{H}^{n+\frac{3}{2}}
\end{array}\right)=\left(\begin{array}{c}
\mathbb{E}^{n} \\
\mathbb{H}^{n+\frac{1}{2}}
\end{array}\right)+\left(2 \sum_{i=1 \text { (odd) }}^{N-1} \frac{1}{i !}\left(\frac{\Delta t}{2}\right)^{i} \mathcal{A}^{i}\right)\left(\begin{array}{c}
\mathbb{E}^{n+1} \\
\mathbb{H}^{n+\frac{1}{2}}
\end{array}\right) .
$$

Note that here, the used time step $\Delta t$ is twice as large as the time step defined in Eq. (2.12). For $N=2$, we recover the second-order DGTD method studied in [8].

The discontinuous Galerkin DGTD- $\mathbb{P}_{p_{i}}$ method using centered fluxes combined with a $N$ th-order leap-frog $\left(\mathrm{LF}_{N}\right)$ time scheme can be written as:

$$
\left\{\begin{array}{l}
\mathbb{M}^{\epsilon} \frac{\mathbb{E}^{n+1}-\mathbb{E}^{n}}{\Delta t}=\mathbb{S}_{N} \mathbb{H}^{n+\frac{1}{2}} \\
\mathbb{M}^{\mu} \frac{\mathbb{H}^{n+\frac{3}{2}}-\mathbb{H}^{n+\frac{1}{2}}}{\Delta t}=-{ }^{t} \mathbb{S}_{N} \mathbb{E}^{n+1}
\end{array}\right.
$$

where the matrix $\mathbb{S}_{N}$ ( $N$ being the order of the leap-frog scheme) verifies:

$$
\mathbb{S}_{N}= \begin{cases}\mathbb{S} & \text { if } N=2, \\ \mathbb{S}\left(\mathbb{I}+\sum_{i=1}^{N / 2-1} \frac{(-1)^{i}}{(2 i+1) ! 2^{2 i}}\left(\Delta t^{2} \mathbb{M}^{-\mu t} \mathbb{S M}^{-\epsilon} \mathbb{S}\right)^{i}\right) & \forall N>2, \text { even }\end{cases}
$$

One can verify that, Eq. (2.19) can be obtained from Eq. (2.17) in a straightforward manner. For instance, taking $N=4$ in Eq. (2.17), yields the $\mathrm{LF}_{4}$ scheme:

$$
\left(\begin{array}{c}
\mathbb{E}^{n+1}-\mathbb{E}^{n} \\
\mathbb{H}^{n+\frac{3}{2}}-\mathbb{H}^{n+\frac{1}{2}}
\end{array}\right)=\left(\begin{array}{cc}
0 & \Delta t \mathbb{M}^{-\epsilon} \mathbb{X} \\
-\Delta t \mathbb{M}^{-\mu t} \mathbb{X} & 0
\end{array}\right)\left(\begin{array}{c}
\mathbb{E}^{n+1} \\
\mathbb{H}^{n+\frac{1}{2}}
\end{array}\right)
$$

where $\mathbb{X}=\mathbb{S}\left(\mathbb{I}-\frac{\Delta t^{2}}{24} \mathbb{M}^{-\mu t} \mathbb{S M}^{-\epsilon} \mathbb{S}\right)=\mathbb{S}_{4}$.

Concerning memory and complexity, the $\mathrm{LF}_{N}$ scheme requires $N / 2$ times more memory storage and $(N-1)$ times more arithmetic operations than the $\mathrm{LF}_{2}$ scheme studied in $[8,9]$. 


\section{Stability and convergence analysis}

In this section we study the stability and convergence properties of the high-order discontinuous Galerkin method introduced previously.

\subsection{Stability}

We aim at giving and proving a sufficient stability condition for the proposed highorder DGTD method, Eqs. (2.18)-(2.19). We use the same kind of energy approach as in [8] where a quadratic form plays the role of a Lyapunov function of the whole set of numerical unknowns. We define the following discrete electromagnetic energy in the whole domain $\Omega$ :

$$
\mathcal{E}^{n}=\frac{1}{2}\left({ }^{t} \mathbb{E}^{n} \mathbb{M}^{\epsilon} \mathbb{E}^{n}+{ }^{t} \mathbb{H}^{n-\frac{1}{2}} \mathbb{M}^{\mu} \mathbb{H}^{n+\frac{1}{2}}\right) .
$$

Lemma 3.1. Using the DGTD- $\mathbb{P}_{p_{i}}$ method, Eqs. (2.18)-(2.19), the global discrete electromagnetic energy $\mathcal{E}^{n}$ given in Eq. (3.1) is a positive definite quadratic form of all unknowns if:

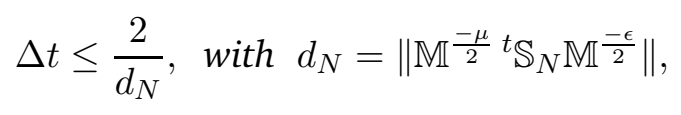

where $\|$.$\| denotes a matrix norm, and the matrix \mathbb{M}^{\frac{-\sigma}{2}}$ is the inverse square root of $\mathbb{M}^{\sigma}$.

Proof. The mass matrices $\mathbb{M}^{\epsilon}$ and $\mathbb{M}^{\mu}$ are symmetric positive definite and we can construct in a simple way their square root (also symmetric positive definite) denoted by $\mathbb{M}^{\frac{\epsilon}{2}}$ and $\mathbb{M}^{\frac{\mu}{2}}$ respectively. Moreover:

$$
\begin{aligned}
2 \mathcal{E}^{n} & ={ }^{t} \mathbb{E}^{n} \mathbb{M}^{\epsilon} \mathbb{E}^{n}+{ }^{t} \mathbb{H}^{n-\frac{1}{2}} \mathbb{M}^{\mu} \mathbb{H}^{n-\frac{1}{2}}-\Delta t \mathbb{H}^{n-\frac{1}{2}} t \mathbb{S}_{N} \mathbb{E}^{n} \\
& \geq\left\|\mathbb{M}^{\frac{\epsilon}{2}} \mathbb{E}^{n}\right\|^{2}+\left\|\mathbb{M}^{\frac{\mu}{2}} \mathbb{H}^{n-\frac{1}{2}}\right\|^{2}-\Delta t\left|\mathbb{H}^{n-\frac{1}{2}} \mathbb{M}^{\frac{\mu}{2}} \mathbb{M}^{\frac{-\mu}{2}} t \mathbb{S}_{N} \mathbb{M}^{\frac{\epsilon}{2}} \mathbb{M} \frac{\epsilon}{2} \mathbb{E}^{n}\right| \\
& \geq\left\|\mathbb{M}^{\frac{\epsilon}{2}} \mathbb{E}^{n}\right\|^{2}+\left\|\mathbb{M}^{\frac{\mu}{2}} \mathbb{H}^{n-\frac{1}{2}}\right\|^{2}-d_{N} \Delta t\left\|\mathbb{M}^{\frac{\mu}{2}} \mathbb{H}^{n-\frac{1}{2}}\right\|\left\|\mathbb{M}^{\frac{\epsilon}{2}} \mathbb{E}^{n}\right\| \\
& \geq\left\|\mathbb{M}^{\frac{\epsilon}{2}} \mathbb{E}^{n}\right\|^{2}+\left\|\mathbb{M}^{\frac{\mu}{2}} \mathbb{H}^{n-\frac{1}{2}}\right\|^{2}-\frac{d_{N} \Delta t}{2}\left(\left\|\mathbb{M}^{\frac{\mu}{2}} \mathbb{H}^{n-\frac{1}{2}}\right\|^{2}+\left\|\mathbb{M}^{\frac{\epsilon}{2}} \mathbb{E}^{n}\right\|^{2}\right) .
\end{aligned}
$$

We then sum up the lower bounds for the $\mathcal{E}^{n}$ to obtain:

$$
2 \mathcal{E}^{n} \geq\left(1-\frac{d_{N} \Delta t}{2}\right)\left\|\mathbb{M}^{\frac{\epsilon}{2}} \mathbb{E}^{n}\right\|^{2}+\left(1-\frac{d_{N} \Delta t}{2}\right)\left\|\mathbb{M}^{\frac{\mu}{2}} \mathbb{H}^{n-\frac{1}{2}}\right\|^{2}
$$

Then, under the condition proposed in Lemma 3.1, the electromagnetic energy $\mathcal{E}$ is a positive definite quadratic form of all unknowns. This concludes the proof.

Now, we denote by $\nu_{N}=\mathrm{CFL}\left(\mathrm{LF}_{N}\right) / \mathrm{CFL}\left(\mathrm{LF}_{2}\right)$ the ratio between the stability limit of the $\mathrm{LF}_{N}$ scheme and the $\mathrm{LF}_{2}$ scheme, and by $r_{N}=\nu_{N} /(N / 2)$ the ratio between $\nu_{N}$ and the additional memory storage between the $\mathrm{LF}_{N}$ and $\mathrm{LF}_{2}$ schemes. Tab. 1 lists the values of $\nu_{N}$ and $r_{N}$ for several values of $N$. As it can be seen from Tab. 1, the choice of the $\mathrm{LF}_{4}$ scheme is advantageous with respect to the $r_{N}$ ratio. 
Table 1: The values of $\nu_{N}$ and $r_{N}$ for several $\mathrm{LF}_{N}$ schemes.

\begin{tabular}{|c|c|c|c|c|c|c|c|c|c|}
\hline$N$ & $\mathbf{4}$ & $\mathbf{6}$ & $\mathbf{8}$ & $\mathbf{1 0}$ & $\mathbf{1 2}$ & $\mathbf{1 4}$ & $\mathbf{1 6}$ & $\mathbf{1 8}$ & $\mathbf{2 0}$ \\
\hline \hline$\nu_{N}$ & 2.847 & 3.681 & 3.793 & 5.272 & 4.437 & 6.422 & 7.534 & 7.265 & 8.909 \\
$r_{N}$ & 1.424 & 1.227 & 0.948 & 1.05 & 0.739 & 0.917 & 0.942 & 0.807 & 0.891 \\
\hline
\end{tabular}

\subsection{Convergence}

In this section, our objective is to obtain an a priori error estimates depending on $h$ and $p$, which establishes the rate of convergence of the proposed $h p$-like DGTD method. We assume that $\overline{\bar{\epsilon}}, \overline{\bar{\mu}} \in\left[L^{\infty}(\Omega)\right]^{3 \times 3}$ and $\exists C_{1}, C_{2}>0$ such that:

$$
\forall \vec{\xi} \in \mathbb{R}^{3}: \quad C_{1}|\vec{\xi}|^{2} \leq \overline{\bar{\epsilon}} \vec{\xi} \cdot \vec{\xi} \leq C_{2}|\vec{\xi}|^{2}, \quad C_{1}|\vec{\xi}|^{2} \leq \overline{\bar{\mu}} \vec{\xi} \cdot \vec{\xi} \leq C_{2}|\vec{\xi}|^{2}
$$

The problem in Eqs. (2.1)-(2.2) admits a unique solution $(\overrightarrow{\mathbf{E}}, \overrightarrow{\mathbf{H}}) \in\left[C^{1}\left(0, T ;\left[L^{2}(\Omega)\right]^{3}\right) \cap\right.$ $\left.C^{0}\left(0, T ; H_{0}(\operatorname{curl}, \Omega)\right)\right]^{2}$ under some regularity assumptions on the initial condition $\overrightarrow{\mathbf{E}}_{0}$ and $\overrightarrow{\mathbf{H}}_{0}$ (see [17]).

For a real $s \geq 0$, we define the classical broken space :

$$
H^{s}\left(\Omega_{h}\right)=\left\{v \in L^{2}(\Omega): \forall \tau_{i} \in \Omega_{h}, v_{\mid \tau_{i}} \in H^{s}\left(\tau_{i}\right)\right\} .
$$

The space $H^{s}\left(\Omega_{h}\right)$ is equipped with the natural norm, for $v \in H^{s}\left(\Omega_{h}\right)$ :

$$
\|v\|_{s, h}=\left(\sum_{\tau_{i} \in \Omega_{h}}\|v\|_{s, \tau_{i}}^{2}\right)^{\frac{1}{2}}
$$

where $\|\cdot\|_{s, \tau_{i}}$ is the usual Sobolev norm of $H^{s}$ on $\tau_{i}$. For $s>1 / 2$, the elementwise traces of functions in $H^{s}\left(\Omega_{h}\right)$ belongs to $\operatorname{tr}\left(\mathcal{F}_{h}\right)=\Pi_{\tau_{i} \in \Omega_{h}} L^{2}\left(\partial \tau_{i}\right)$. We denote by $\mathbf{H}^{s}\left(\Omega_{h}\right)$ the vectorial broken space $\left[H^{s}\left(\Omega_{h}\right)\right]^{3}$ and the associated norm defined by :

$$
\|\vec{v}\|_{s, h}=\left(\sum_{j=1}^{3}\left\|v_{j}\right\|_{s, h}^{2}\right)^{\frac{1}{2}}
$$

where $\vec{v}=\left(v_{1}, v_{2}, v_{3}\right) \in \mathbf{H}^{s}\left(\Omega_{h}\right)$. We define the jump of a function $\vec{v} \in \mathbf{H}^{s}\left(\Omega_{h}\right)$ :

$$
\begin{aligned}
& \forall a_{i k} \in \mathcal{F}_{h}^{I}, \quad \llbracket \vec{v} \rrbracket_{i k}^{i}=\llbracket \vec{v} \rrbracket_{a_{i k}}^{\tau_{i}}=\left(\vec{v}_{k \mid a_{i k}}-\vec{v}_{i \mid a_{i k}}\right) \times \vec{n}_{i k}, \\
& \forall a_{i k} \in \mathcal{F}_{h}^{B}, \quad \llbracket \vec{v} \rrbracket_{i k}^{i}=-\vec{v}_{i \mid a_{i k}} \times \vec{n}_{i k} .
\end{aligned}
$$

We associate to the continuous problem in Eq. (2.1) the following space discretized problem: find $(\overrightarrow{\mathbf{E}}(., t), \overrightarrow{\mathbf{H}}(., t)) \in \mathbf{H}^{1}\left(\Omega_{h}\right) \times \mathbf{H}^{1}\left(\Omega_{h}\right)$ such that, $\forall \tau_{i} \in \Omega_{h}$ and $\forall \vec{\phi}, \vec{\psi} \in$ $\mathbf{H}^{1}\left(\Omega_{h}\right)$, 


$$
\left\{\begin{aligned}
\int_{\tau_{i}} \vec{\phi}_{i} \cdot \overline{\bar{\epsilon}}_{i} \partial_{t} \overrightarrow{\mathbf{E}}_{i}-\int_{\tau_{i}} \overrightarrow{\mathbf{H}}_{i} \cdot \operatorname{curl} \vec{\phi}_{i} & +\sum_{\substack{k \in \mathcal{V}_{i} \\
a_{i k} \in \mathcal{F}_{h}^{I}}} \int_{a_{i k}} \vec{\phi}_{i} \cdot\left(\overrightarrow{\mathbf{H}}_{\mid a_{i k}} \times \vec{n}_{i k}\right) \\
& +\sum_{\substack{k \in \mathcal{V}_{i} \\
a_{i k} \in \mathcal{F}_{h}^{B}}} \int_{a_{i k}} \vec{\phi}_{i} \cdot\left(\overrightarrow{\mathbf{H}}_{\mid a_{i k}} \times \vec{n}_{i k}\right)=0 \\
\int_{\tau_{i}} \vec{\psi}_{i} \cdot \overline{\bar{\mu}}_{i} \partial_{t} \overrightarrow{\mathbf{H}}_{i}+\int_{\tau_{i}} \overrightarrow{\mathbf{E}}_{i} \cdot \operatorname{curl} \vec{\psi}_{i}-\sum_{\substack{k \in \mathcal{V}_{i} \\
a_{i k} \in \mathcal{F}_{h}^{I}}} \int_{a_{i k}} \vec{\psi}_{i} \cdot\left(\overrightarrow{\mathbf{E}}_{\mid a_{i k}} \times \vec{n}_{i k}\right) & =0
\end{aligned}\right.
$$

where $\vec{\phi}_{i}=\vec{\phi}_{\mid \tau_{i}}$ and $\vec{\psi}_{i}=\vec{\psi}_{\mid \tau_{i}}$. Summing up the identities in Eq. (3.8) with respect to $i$, we consider the following semi-discrete discontinuous Galerkin problem: find $\left(\overrightarrow{\mathbf{E}}_{h}(., t), \overrightarrow{\mathbf{H}}_{h}(., t)\right) \in V_{p}\left(\Omega_{h}\right) \times V_{p}\left(\Omega_{h}\right)$ such that, $\forall \tau_{i} \in \Omega_{h}$ and $\forall \vec{\phi}_{h}, \vec{\psi}_{h} \in V_{p}\left(\Omega_{h}\right)$,

$$
\left\{\begin{array}{l}
\sum_{i} \int_{\tau_{i}} \vec{\phi}_{h i} \cdot \overline{\bar{\epsilon}}_{i} \partial_{t} \overrightarrow{\mathbf{E}}_{i}-\sum_{i} \int_{\tau_{i}} \overrightarrow{\mathbf{H}}_{i} \cdot \operatorname{curl} \vec{\phi}_{h i}+\sum_{a_{i k} \in \mathcal{F}_{h}} \int_{a_{i k}} \llbracket \vec{\phi}_{h} \rrbracket_{i k}^{i} \cdot\left\{\overrightarrow{\mathbf{H}}_{h}\right\}_{i k}=0 \\
\sum_{i} \int_{\tau_{i}} \vec{\psi}_{h i} \cdot \overline{\bar{\mu}}_{i} \partial_{t} \overrightarrow{\mathbf{H}}_{i}+\sum_{i} \int_{\tau_{i}} \overrightarrow{\mathbf{E}}_{i} \cdot \operatorname{curl} \vec{\psi}_{h i}-\sum_{a_{i k} \in \mathcal{F}_{h}} \int_{a_{i k}} \llbracket \vec{\psi}_{h} \rrbracket_{i k}^{i} \cdot\left\{\overrightarrow{\mathbf{E}}_{h}\right\}_{i k}=0 \\
\overrightarrow{\mathbf{E}}_{h}(0)=\Pi_{h}^{p} \overrightarrow{\mathbf{E}}_{0} \text { and } \overrightarrow{\mathbf{H}}_{h}(0)=\Pi_{h}^{p} \overrightarrow{\mathbf{H}}_{0} .
\end{array}\right.
$$

Here $\Pi_{h}^{p}: \mathbf{L}^{2}(\Omega) \rightarrow V_{p}\left(\Omega_{h}\right)$ is the $\mathbf{L}^{2}$-orthogonal projection onto $V_{p}\left(\Omega_{h}\right)$. The problem (3.9) can be rewritten in the following form: find $\overrightarrow{\mathbf{U}}_{h}=\left(\overrightarrow{\mathbf{E}}_{h}, \overrightarrow{\mathbf{H}}_{h}\right) \in V_{p}\left(\Omega_{h}\right) \times V_{p}\left(\Omega_{h}\right)$ such that:

$$
J\left(\partial_{t} \overrightarrow{\mathbf{U}}_{h}, \overrightarrow{\mathbf{U}}^{\prime}{ }_{h}\right)+a\left(\overrightarrow{\mathbf{U}}_{h}, \overrightarrow{\mathbf{U}}^{\prime}{ }_{h}\right)+b\left(\overrightarrow{\mathbf{U}}_{h}, \overrightarrow{\mathbf{U}}^{\prime}{ }_{h}\right)=0, \forall \overrightarrow{\mathbf{U}}_{h}^{\prime} \in V_{p}\left(\Omega_{h}\right) \times V_{p}\left(\Omega_{h}\right) .
$$

For $\overrightarrow{\mathbf{W}}=(\vec{u}, \vec{v})$ and $\overrightarrow{\mathbf{W}}^{\prime}=\left(\overrightarrow{u^{\prime}}, \overrightarrow{v^{\prime}}\right)$, the bilinear forms $J, a$ and $b$ defined on $V_{p}\left(\Omega_{h}\right) \times$ $V_{p}\left(\Omega_{h}\right)$ are given by:

$$
\left\{\begin{aligned}
J\left(\overrightarrow{\mathbf{W}}, \overrightarrow{\mathbf{W}}^{\prime}\right) & =\sum_{i} \int_{\tau_{i}}\left(\overline{\bar{\epsilon}} \vec{u} \cdot \overrightarrow{u^{\prime}}+\overline{\bar{\mu}} \vec{v} \cdot \overrightarrow{v^{\prime}}\right) \\
a\left(\overrightarrow{\mathbf{W}}, \overrightarrow{\mathbf{W}}^{\prime}\right) & =\sum_{i} \int_{\tau_{i}}\left(\vec{u} \cdot \operatorname{curl}_{h} \overrightarrow{v^{\prime}}-\vec{v} \cdot \operatorname{curl}_{h} \overrightarrow{u^{\prime}}\right) \\
b\left(\overrightarrow{\mathbf{W}}, \overrightarrow{\mathbf{W}}^{\prime}\right) & =\sum_{a_{i k} \in \mathcal{F}_{h}} \int_{a_{i k}}\left(\{\vec{v}\} \cdot \llbracket \overrightarrow{u^{\prime}} \rrbracket-\{\vec{u}\} \cdot \llbracket \overrightarrow{v^{\prime}} \rrbracket\right)
\end{aligned}\right.
$$

taking into account that, for boundary faces $a_{i k} \in \mathcal{F}_{h}^{B}$ we have $\{\vec{v}\}=\vec{v}$. Here, $\operatorname{curl}_{h}$ is the piecewise curl-operator given by $\forall i,\left(\operatorname{curl}_{h} \vec{u}\right)_{\mid \tau_{i}}=\operatorname{curl}\left(\vec{u}_{\mid \tau_{i}}\right)$. The semi-discrete 
discontinuous Galerkin formulation, Eq. (3.10), is consistent with the original continuous problem, Eq. (2.1), in the following sense: if $\overrightarrow{\mathbf{U}}=(\overrightarrow{\mathbf{E}}, \overrightarrow{\mathbf{H}})$ is the exact solution of Eq. (2.1), such that $\forall t \in[0, T], \overrightarrow{\mathbf{U}}(., t) \in \mathbf{H}^{s}(\Omega) \times \mathbf{H}^{s}(\Omega)$, then we have:

$$
J\left(\partial_{t} \overrightarrow{\mathbf{U}}, \overrightarrow{\mathbf{U}}^{\prime}\right)+a\left(\overrightarrow{\mathbf{U}}, \overrightarrow{\mathbf{U}}^{\prime}\right)+b\left(\overrightarrow{\mathbf{U}}, \overrightarrow{\mathbf{U}}^{\prime}\right)=0, \quad \forall \overrightarrow{\mathbf{U}}^{\prime} \in V_{p}\left(\Omega_{h}\right) \times V_{p}\left(\Omega_{h}\right) .
$$

The following approximation results will be used to bound the error $[2,19]$.

Lemma 3.2. (Babuska and Suri [2]) Let $\tau_{i} \in \Omega_{h}$ and suppose that $\vec{u} \in \mathbf{H}^{s}\left(\tau_{i}\right), s \geq 1 / 2$. Let $\Pi$ be a linear continuous operator from $\mathbf{H}^{s}\left(\tau_{i}\right)$ onto $\mathbb{P}_{p_{i}}\left(\tau_{i}\right)$, $p_{i} \geq 1$, such that $\Pi(\vec{u})=$ $\vec{u}, \forall \vec{u} \in \mathbb{P}_{p_{i}}\left(\tau_{i}\right)$. Then we have:

$$
\begin{aligned}
\|\vec{u}-\Pi(\vec{u})\|_{s^{\prime}, \tau_{i}} & \leq C \frac{h_{i}^{\nu_{i}-s^{\prime}}}{p_{i}^{s-s^{\prime}}}\|\vec{u}\|_{s, \tau_{i}}, \\
\|\vec{u}-\Pi(\vec{u})\|_{0, \partial \tau_{i}} & \leq C \frac{h_{i}^{\nu_{i}-1 / 2}}{p_{i}^{s-1 / 2}}\|\vec{u}\|_{s, \tau_{i}},
\end{aligned}
$$

where $\nu_{i}=\min \left\{s, p_{i}+1\right\}, 0 \leq s^{\prime} \leq \nu_{i}$, and $C$ is a positive constant independent of $u, h_{i}$ and $p_{i}$, but dependent on $s$ and on the shape regularity of the mesh parameter $\eta$.

Lemma 3.3. (Schwab [19]) For all $q \in \mathbb{P}_{p_{i}}\left(\tau_{i}\right), p_{i} \geq 1$, we have:

$$
\|q\|_{0, \partial \tau_{i}}^{2} \leq C_{i n v} \frac{p_{i}^{2}}{h_{i}}\|q\|_{0, \tau_{i}}^{2}
$$

where $C_{\text {inv }}$ is a positive constant depending only on the shape regularity of the mesh parameter $\eta$.

Let $\overrightarrow{\mathbf{U}}=(\overrightarrow{\mathbf{E}}, \overrightarrow{\mathbf{H}})$ and $\overrightarrow{\mathbf{U}}_{h}=\left(\overrightarrow{\mathbf{E}}_{h}, \overrightarrow{\mathbf{H}}_{h}\right)$. We define $\boldsymbol{\Pi}_{h}^{p}: \mathbf{L}^{2}(\Omega) \times \mathbf{L}^{2}(\Omega) \rightarrow V_{p}\left(\Omega_{h}\right) \times$ $V_{p}\left(\Omega_{h}\right)$ by $\Pi_{h}^{p}(\overrightarrow{\mathbf{U}})=\left(\Pi_{h}^{p} \overrightarrow{\mathbf{E}}, \Pi_{h}^{p} \overrightarrow{\mathbf{H}}\right)$. We denote by $\varepsilon_{\tau_{i}}(t)$ the local error and by $\varepsilon(t)=$ $\sum_{\tau_{i} \in \Omega_{h}} \boldsymbol{\varepsilon}_{\tau_{i}}(t)$ the global error. Then we have:

$$
\begin{aligned}
\boldsymbol{\varepsilon}_{\tau_{i}}(t) & =\left\|\overrightarrow{\mathbf{E}}-\Pi_{h}^{p} \overrightarrow{\mathbf{E}}+\Pi_{h}^{p} \overrightarrow{\mathbf{E}}-\overrightarrow{\mathbf{E}}_{h}\right\|_{0, \tau_{i}}^{2}+\left\|\overrightarrow{\mathbf{H}}-\Pi_{h}^{p} \overrightarrow{\mathbf{H}}+\Pi_{h}^{p} \overrightarrow{\mathbf{H}}-\overrightarrow{\mathbf{H}}_{h}\right\|_{0, \tau_{i}}^{2} \\
& \leq 2\left\|\overrightarrow{\mathbf{U}}-\Pi_{h}^{p} \overrightarrow{\mathbf{U}}\right\|_{0, \tau_{i}}^{2}+2\left\|\Pi_{h}^{p} \overrightarrow{\mathbf{U}}-\overrightarrow{\mathbf{U}}_{h}\right\|_{0, \tau_{i}}^{2} \\
& =2 \varepsilon_{\tau_{i}}^{a}+2 \varepsilon_{\tau_{i}}^{b},
\end{aligned}
$$

where $\varepsilon_{\tau_{i}}^{a}$ is due to the error introduced by the polynomial approximation of the exact solution while $\varepsilon_{\tau_{i}}^{b}$ measures the errors associated with the semi-discrete approximation of Maxwell's equations.

To bound $\varepsilon_{\tau_{i}}^{a}$ we need only recall Lemma 3.2 to state: 
Lemma 3.4. Assume that $\overrightarrow{\mathbf{U}} \in \mathbf{H}^{s}\left(\tau_{i}\right) \times \mathbf{H}^{s}\left(\tau_{i}\right), s \geq 0$. Then there exists a constant $C$, dependent on $s$ and on the shape regularity of the mesh $\eta$, but independent of $\overrightarrow{\mathbf{U}}, h_{i}$ and $p_{i}$, such that:

$$
\left\|\overrightarrow{\mathbf{U}}-\boldsymbol{\Pi}_{h}^{p} \overrightarrow{\mathbf{U}}\right\|_{0, \tau_{i}} \leq C \frac{h_{i}^{\nu_{i}}}{p_{i}^{s}}\|\overrightarrow{\mathbf{U}}\|_{s, \tau_{i}},
$$

where $\nu_{i}=\min \left\{s, p_{i}+1\right\}$.

Theorem 3.1. Assume that a solution $(\overrightarrow{\mathbf{E}}(t), \overrightarrow{\mathbf{H}}(t)) \in \mathbf{H}^{s}\left(\tau_{i}\right) \times \mathbf{H}^{s}\left(\tau_{i}\right)$ with $s \geq 3 / 2$ to Maxwell's equations in $\Omega_{h}=\bigcup_{i} \tau_{i}$ exists. Then the numerical solution, $\left(\overrightarrow{\mathbf{E}}_{h}(t), \overrightarrow{\mathbf{H}}_{h}(t)\right) \in$ $V_{p}\left(\Omega_{h}\right) \times V_{p}\left(\Omega_{h}\right)$, to the semi-discrete approximation, Eq. (3.9), converges to the exact solution and the global error is bounded as:

$$
\left(\left\|\overrightarrow{\mathbf{E}}-\overrightarrow{\mathbf{E}}_{h}\right\|_{0, \Omega}^{2}+\left\|\overrightarrow{\mathbf{H}}-\overrightarrow{\mathbf{H}}_{h}\right\|_{0, \Omega}^{2}\right)^{\frac{1}{2}} \leq C\left(\frac{h^{\nu}}{p_{\min }^{s}}+T \frac{h^{\nu-1}}{p_{\min }^{s-\frac{3}{2}}}\right) \max _{t \in[0, T]}\|(\overrightarrow{\mathbf{E}}(t), \overrightarrow{\mathbf{H}}(t))\|_{s, \Omega},
$$

where $\nu=\min \left\{s, p_{\min }+1\right\}$ and $p_{\min }=\min \left\{p_{i}, \tau_{i} \in \Omega_{h}\right\}, p_{i} \geq 1$. The constant $C>0$ depends on the material properties and on the shape regularity of the mesh parameter $\eta$, but not on $p_{\min }$ and $h$.

Proof. Let $\overrightarrow{\mathbf{q}}=\overrightarrow{\mathbf{U}}-\overrightarrow{\mathbf{U}}_{h}$. Since $\boldsymbol{\Pi}_{h}^{p} \overrightarrow{\mathbf{U}}_{h}=\overrightarrow{\mathbf{U}}_{h}$, we have $\sum_{i} \varepsilon_{\tau_{i}}^{b}=\left\|\boldsymbol{\Pi}_{h}^{p} \overrightarrow{\mathbf{q}}\right\|_{0, \Omega}^{2}$. To obtain a bound for $\left\|\boldsymbol{\Pi}_{h}^{p} \overrightarrow{\mathbf{q}}\right\|_{0, \Omega}$, we introduce $\sigma(t)=\frac{1}{2} J\left(\boldsymbol{\Pi}_{h}^{p} \overrightarrow{\mathbf{q}}(t), \boldsymbol{\Pi}_{h}^{p} \overrightarrow{\mathbf{q}}(t)\right)$ with $\boldsymbol{\Pi}_{h}^{p} \overrightarrow{\mathbf{q}}(., t)$ belongs to $V_{p}\left(\Omega_{h}\right) \times V_{p}\left(\Omega_{h}\right)$. Using the discrete initial conditions of Eq. (3.9), we have $\sigma(0)=0$ and then, for $0<t \leq T$,

$$
\sigma(t)=\frac{1}{2} \int_{0}^{t} \frac{d}{d s} J\left(\boldsymbol{\Pi}_{h}^{p} \overrightarrow{\mathbf{q}}(s), \boldsymbol{\Pi}_{h}^{p} \overrightarrow{\mathbf{q}}(s)\right) d s=\int_{0}^{t} J\left(\partial_{s} \boldsymbol{\Pi}_{h}^{p} \overrightarrow{\mathbf{q}}(s), \boldsymbol{\Pi}_{h}^{p} \overrightarrow{\mathbf{q}}(s)\right) d s .
$$

For any $\overrightarrow{\mathbf{U}}_{h} \in V_{p}\left(\Omega_{h}\right) \times V_{p}\left(\Omega_{h}\right)$, we have $a\left(\overrightarrow{\mathbf{U}}_{h}, \overrightarrow{\mathbf{U}}_{h}\right)+b\left(\overrightarrow{\mathbf{U}}_{h}, \overrightarrow{\mathbf{U}}_{h}\right)=0$, and we get:

$$
\begin{aligned}
\sigma(t)=\int_{0}^{t}\left(J\left(\partial_{s} \boldsymbol{\Pi}_{h}^{p} \overrightarrow{\mathbf{q}}(s), \boldsymbol{\Pi}_{h}^{p} \overrightarrow{\mathbf{q}}(s)\right)+\right. & a\left(\boldsymbol{\Pi}_{h}^{p} \overrightarrow{\mathbf{q}}(s), \boldsymbol{\Pi}_{h}^{p} \overrightarrow{\mathbf{q}}(s)\right) \\
& \left.+b\left(\boldsymbol{\Pi}_{h}^{p} \overrightarrow{\mathbf{q}}(s), \boldsymbol{\Pi}_{h}^{p} \overrightarrow{\mathbf{q}}(s)\right)\right) d s .
\end{aligned}
$$

Subtracting Eq. (3.10) from the consistency result of Eq. (3.12) with $\overrightarrow{\mathbf{U}}^{\prime}=\overrightarrow{\mathbf{U}}_{h}^{\prime}=$ $\boldsymbol{\Pi}_{h}^{p} \overrightarrow{\mathbf{q}}(s)$ yields:

$$
J\left(\partial_{s} \overrightarrow{\mathbf{q}}(s), \boldsymbol{\Pi}_{h}^{p} \overrightarrow{\mathbf{q}}(s)\right)+a\left(\overrightarrow{\mathbf{q}}(s), \boldsymbol{\Pi}_{h}^{p} \overrightarrow{\mathbf{q}}(s)\right)+b\left(\overrightarrow{\mathbf{q}}(s), \boldsymbol{\Pi}_{h}^{p} \overrightarrow{\mathbf{q}}(s)\right)=0 .
$$

Now, subtracting the above equality in Eq. (3.18) from Eq. (3.17) leads to:

$$
\begin{aligned}
\sigma(t)=\int_{0}^{t}\left(J\left(\left[\boldsymbol{\Pi}_{h}^{p} \partial_{s} \overrightarrow{\mathbf{U}}-\partial_{s} \overrightarrow{\mathbf{U}}\right](s), \boldsymbol{\Pi}_{h}^{p} \overrightarrow{\mathbf{q}}(s)\right)\right. & +a\left(\left[\boldsymbol{\Pi}_{h}^{p} \overrightarrow{\mathbf{U}}-\overrightarrow{\mathbf{U}}\right](s), \boldsymbol{\Pi}_{h}^{p} \overrightarrow{\mathbf{q}}(s)\right) \\
& \left.+b\left(\left[\boldsymbol{\Pi}_{h}^{p} \overrightarrow{\mathbf{U}}-\overrightarrow{\mathbf{U}}\right](s), \boldsymbol{\Pi}_{h}^{p} \overrightarrow{\mathbf{q}}(s)\right)\right) d s .
\end{aligned}
$$


Since $\boldsymbol{\Pi}_{h}^{p}$ is a projector onto $V_{p}\left(\Omega_{h}\right) \times V_{p}\left(\Omega_{h}\right)$ and $\boldsymbol{\Pi}_{h}^{p} \overrightarrow{\mathbf{q}}(., t)$ belongs to $V_{p}\left(\Omega_{h}\right) \times V_{p}\left(\Omega_{h}\right)$, then $J\left(\boldsymbol{\Pi}_{h}^{p} \partial_{s} \overrightarrow{\mathbf{U}}-\partial_{s} \overrightarrow{\mathbf{U}}, \boldsymbol{\Pi}_{h}^{p} \overrightarrow{\mathbf{q}}\right)=0$. In the same way, it follows that $a\left(\boldsymbol{\Pi}_{h}^{p} \overrightarrow{\mathbf{U}}-\overrightarrow{\mathbf{U}}, \boldsymbol{\Pi}_{h}^{p} \overrightarrow{\mathbf{q}}\right)=0$ since $\operatorname{curl}_{h}\left(\boldsymbol{\Pi}_{h}^{p} \overrightarrow{\mathbf{q}}\right)(s) \in V_{p}\left(\Omega_{h}\right) \times V_{p}\left(\Omega_{h}\right)$ for all $0<s \leq t$. Using the lower bound $C_{1}>0$ of $\overline{\bar{\epsilon}}$ and $\overline{\bar{\mu}}$, Eq. (3.3), we thus get:

$$
\frac{C_{1}}{2}\left\|\boldsymbol{\Pi}_{h}^{p} \overrightarrow{\mathbf{q}}(t)\right\|_{0, \Omega}^{2} \leq \int_{0}^{t} b\left(\left[\boldsymbol{\Pi}_{h}^{p} \overrightarrow{\mathbf{U}}-\overrightarrow{\mathbf{U}}\right](s), \boldsymbol{\Pi}_{h}^{p} \overrightarrow{\mathbf{q}}(s)\right) d s .
$$

Now, we bound the surface integrals deriving from the definition of $b(.,$.$) . We assume$ that $\overrightarrow{\mathbf{q}}=\left(\overrightarrow{\mathbf{q}}^{E}, \overrightarrow{\mathbf{q}}^{H}\right)$, where $\overrightarrow{\mathbf{q}}^{E}$ and $\overrightarrow{\mathbf{q}}^{H}$ denote the error in $\overrightarrow{\mathbf{E}}$ and $\overrightarrow{\mathbf{H}}$ respectively. Let $a_{i k} \in \mathcal{F}_{h}^{I}$ be an internal interface shared by the tetrahedra $\tau_{i}$ and $\tau_{k}$. We denote by $\mathbb{I}^{E}=\int_{a_{i k}}\left\{\Pi_{h}^{p} \overrightarrow{\mathbf{H}}-\overrightarrow{\mathbf{H}}\right\}_{i k} \cdot \llbracket \Pi_{h}^{p} \overrightarrow{\mathbf{q}}^{E} \rrbracket_{i k}$, we have, using the Cauchy-Schwarz-Buniakovsky (CSB) inequality:

$$
\mathbb{I}^{E} \leq \underbrace{\left(\int_{a_{i k}}\left(\left\{\Pi_{h}^{p} \overrightarrow{\mathbf{H}}-\overrightarrow{\mathbf{H}}\right\}_{i k}\right)^{2}\right)^{\frac{1}{2}}}_{\mathbb{I}_{1}^{E}} \underbrace{\left(\int_{a_{i k}}\left(\llbracket \Pi_{h}^{p} \overrightarrow{\mathbf{q}}^{E} \rrbracket_{i k}\right)^{2}\right)^{\frac{1}{2}}}_{\mathbb{I}_{2}^{E}} .
$$

We have that:

$$
\begin{aligned}
\mathbb{I}_{1}^{E} & \leq \frac{\sqrt{2}}{2}\left(\left\|\Pi_{h}^{p} \overrightarrow{\mathbf{H}}_{i}-\overrightarrow{\mathbf{H}}_{i}\right\|_{0, a_{i k}}^{2}+\left\|\Pi_{h}^{p} \overrightarrow{\mathbf{H}}_{k}-\overrightarrow{\mathbf{H}}_{k}\right\|_{0, a_{i k}}^{2}\right)^{\frac{1}{2}} \\
\mathbb{I}_{2}^{E} & \leq \sqrt{2}\left(\left\|\left(\boldsymbol{\Pi}_{h}^{p} \overrightarrow{\mathbf{q}}^{E}\right)_{i}\right\|_{0, a_{i k}}^{2}+\left\|\left(\boldsymbol{\Pi}_{h}^{p} \overrightarrow{\mathbf{q}}^{E}\right)_{k}\right\|_{0, a_{i k}}^{2}\right)^{\frac{1}{2}} .
\end{aligned}
$$

Using Lemma 3.2 and Lemma 3.3, yields:

$$
\mathbb{I}^{E} \leq C\left(\left(\frac{h_{i}^{\nu_{i}-\frac{1}{2}}}{p_{i}^{s-\frac{1}{2}}}\right)^{2}\|\overrightarrow{\mathbf{H}}\|_{s, \tau_{i}}^{2}+\left(\frac{h_{k}^{\nu_{k}-\frac{1}{2}}}{p_{k}^{s-\frac{1}{2}}}\right)^{2}\|\overrightarrow{\mathbf{H}}\|_{s, \tau_{k}}^{2}\right)^{\frac{1}{2}}\left(\frac{p_{i}^{2}}{h_{i}}\left\|\mathbf{\Pi}_{h}^{p} \overrightarrow{\mathbf{q}}^{E}\right\|_{0, \tau_{i}}^{2}+\frac{p_{k}^{2}}{h_{k}}\left\|\boldsymbol{\Pi}_{h}^{p} \overrightarrow{\mathbf{q}}^{E}\right\|_{0, \tau_{k}}^{2}\right)^{\frac{1}{2}}
$$

According to the assumptions of Eq. (2.3), we finally get:

$$
\mathbb{I}^{E} \leq K\left(\kappa_{1}, \kappa_{2}\right) \frac{h_{i}^{\nu-1}}{p_{i}^{s-\frac{3}{2}}}\left(\|\overrightarrow{\mathbf{H}}\|_{s, \tau_{i}}^{2}+\|\overrightarrow{\mathbf{H}}\|_{s, \tau_{k}}^{2}\right)^{\frac{1}{2}}\left(\left\|\boldsymbol{\Pi}_{h}^{p} \overrightarrow{\mathbf{q}}^{E}\right\|_{0, \tau_{i}}^{2}+\left\|\boldsymbol{\Pi}_{h}^{p} \overrightarrow{\mathbf{q}}^{E}\right\|_{0, \tau_{k}}^{2}\right)^{\frac{1}{2}}
$$

where $K>0$ does not depend on $h_{i}$ and $p_{i}$, but depends on $\kappa_{1}$ and $\kappa_{2}$, and on the local material properties $\left(\overline{\bar{\epsilon}}_{i / k}, \overline{\bar{\mu}}_{i / k}\right)$ associated to $\tau_{i}$ and $\tau_{k}$.

The term $\mathbb{I}^{H}=\int_{a_{i k}}\left\{\Pi_{h}^{p} \overrightarrow{\mathbf{E}}-\overrightarrow{\mathbf{E}}\right\}_{i k} \cdot \llbracket \boldsymbol{\Pi}_{h}^{p} \overrightarrow{\mathbf{q}}^{H} \rrbracket_{i k}$ is treated in the same way, yielding the result:

$$
\mathbb{I}^{H} \leq K\left(\kappa_{1}, \kappa_{2}\right) \frac{h_{i}^{\nu-1}}{p_{i}^{s-\frac{3}{2}}}\left(\|\overrightarrow{\mathbf{E}}\|_{s, \tau_{i}}^{2}+\|\overrightarrow{\mathbf{E}}\|_{s, \tau_{k}}^{2}\right)^{\frac{1}{2}}\left(\left\|\mathbf{\Pi}_{h}^{p} \overrightarrow{\mathbf{q}}^{H}\right\|_{0, \tau_{i}}^{2}+\left\|\boldsymbol{\Pi}_{h}^{p} \overrightarrow{\mathbf{q}}^{H}\right\|_{0, \tau_{k}}^{2}\right)^{\frac{1}{2}}
$$


For boundary interfaces $a_{i k} \in \mathcal{F}_{h}^{B}$, we obtain the same upper bounds as Eqs. (3.20) and (3.21) but without the norms on $\tau_{k}$.

Summing up with respect to all $\tau_{i} \in \Omega_{h}$, and using the CSB inequality, yields:

$$
b\left(\left[\boldsymbol{\Pi}_{h}^{p} \overrightarrow{\mathbf{U}}-\overrightarrow{\mathbf{U}}\right](s), \boldsymbol{\Pi}_{h}^{p} \overrightarrow{\mathbf{q}}(s)\right) \leq K\left(\kappa_{1}, \kappa_{2}\right) \frac{h^{\nu-1}}{p_{\min }^{s-\frac{3}{2}}}\left\|\boldsymbol{\Pi}_{h}^{p} \overrightarrow{\mathbf{q}}(s)\right\|_{0, \Omega}\|(\overrightarrow{\mathbf{E}}(s), \overrightarrow{\mathbf{H}}(s))\|_{s, \Omega} .
$$

Integrating in $t \in[0, T]$ and combining this with Lemma 3.4 establishes the result and proves convergence on weak assumptions of local, elementwise smoothness of the solution.

We have hence established the semi-discrete result that the error cannot grow faster than linearly in time and that we can control the growth rate by adapting the resolution parameters $h$ and $p$ accordingly. As we shall verify in Sec. 4 this linear growth is a sharp result. However, the numerical experiments will also show that we can expect that the growth rate approaches zero spectrally fast when increasing the approximation order $p$ provided that the solution is sufficiently smooth.

Note that the convergence result of Theorem 3.1 is different from the one obtained by Fezoui et al. [10]. The convergence result in [10] considers only the case of a conforming discontinuous Galerkin formulation where the interpolation degree is constant. The result presented here remains valid on any kind of mesh and discontinuous elements, including $h p$-type or non-conformal refinement.

\subsection{Convergence of the divergence error}

In the absence of sources, it is well known that the electric and the magnetic fields must remain solenoidal throughout the computation. Indeed, taking the divergence of Eq. (2.1) and applying Eq. (2.2) in combination with Gauss' law for charge conservation immediately confirms that if the initial conditions satisfy Eq. (2.2), and the fields are evolved according to Maxwell's equations Eq. (2.1), the solution will satisfy Eq. (2.2) at all times. Hence, one can view Eq. (2.2) as a consistency condition on the initial conditions and limit the solution to the time-dependent part of Maxwell's equations, Eq. (2.1). The scheme in Eq. (2.7) does not solve Eq. (2.1), however, but rather an approximation to it. Hence, one needs to consider the question of how well Eq. (2.7) conserves the divergence.

Using the results of Sec. 3.2 we can state the following result.

Theorem 3.2. Assume that a solution $\overrightarrow{\mathbf{U}}=(\overrightarrow{\mathbf{E}}(t), \overrightarrow{\mathbf{H}}(t)) \in \mathbf{H}^{s}\left(\tau_{i}\right) \times \mathbf{H}^{s}\left(\tau_{i}\right)$ with $s \geq 7 / 2$ to Maxwell's equations in $\Omega_{h}=\bigcup_{i} \tau_{i}$ exists. Then there exist a constant $C$ dependent on $s$ and the shape regularity of the mesh parameter $\eta$, but independent of $\overrightarrow{\mathbf{U}}, h$, and $p$, such that the divergence of the numerical solution, $\overrightarrow{\mathbf{U}}_{h}$, to the semi-discrete approximation, Eq. (3.9), is bounded as: 


$$
\begin{aligned}
\left(\left\|\nabla \cdot\left(\overrightarrow{\mathbf{E}}-\overrightarrow{\mathbf{E}}_{h}\right)\right\|_{0, \Omega}^{2}+\right. & \left.\left\|\nabla \cdot\left(\overrightarrow{\mathbf{H}}-\overrightarrow{\mathbf{H}}_{h}\right)\right\|_{0, \Omega}^{2}\right)^{\frac{1}{2}} \leq \\
& C\left(\frac{h^{\nu-1}}{p_{\min }^{s-1}}+T \frac{h^{\nu-2}}{p_{\min }^{s-\frac{7}{2}}}\right) \max _{t \in[0, T]}\|(\overrightarrow{\mathbf{E}}(t), \overrightarrow{\mathbf{H}}(t))\|_{s, \Omega},
\end{aligned}
$$

where $\nu=\min \left\{s, p_{\min }+1\right\}$ and $p_{\min }=\min \left\{p_{i}, \tau_{i} \in \Omega_{h}\right\}, p_{i} \geq 1$.

Proof. Consider the local divergence of $\overrightarrow{\mathbf{H}}$ on any $\tau_{i} \in \Omega_{h}$ we have:

$$
\left\|\nabla \cdot\left(\overrightarrow{\mathbf{H}}-\overrightarrow{\mathbf{H}}_{h}\right)\right\|_{0, \tau_{i}}^{2} \leq 2\left\|\nabla \cdot\left(\overrightarrow{\mathbf{H}}-\Pi_{h}^{p} \overrightarrow{\mathbf{H}}\right)\right\|_{0, \tau_{i}}^{2}+2\left\|\nabla \cdot\left(\Pi_{h}^{p} \overrightarrow{\mathbf{H}}-\overrightarrow{\mathbf{H}}_{h}\right)\right\|_{0, \tau_{i}}^{2}
$$

The first term can be bounded using Lemma 3.2 as:

$$
\left\|\nabla \cdot\left(\overrightarrow{\mathbf{H}}-\Pi_{h}^{p} \overrightarrow{\mathbf{H}}\right)\right\|_{0, \tau_{i}} \leq C\left\|\overrightarrow{\mathbf{H}}-\Pi_{h}^{p} \overrightarrow{\mathbf{H}}\right\|_{1, \tau_{i}} \leq C \frac{h_{i}^{\nu_{i}-1}}{p_{i}^{s-1}}\|\overrightarrow{\mathbf{H}}\|_{s, \tau_{i}},
$$

where $\nu_{i}=\min \left\{s, p_{i}+1\right\}$ and $s \geq 1$.

Using the inverse inequality [19]:

$$
\left\|\nabla \cdot \overrightarrow{\mathbf{u}}_{h}\right\|_{0, \tau_{i}} \leq C \frac{p_{i}^{2}}{h_{i}}\left\|\overrightarrow{\mathbf{u}}_{h}\right\|_{0, \tau_{i}}
$$

for all $\overrightarrow{\mathbf{u}}_{h} \in \mathbb{P}_{p_{i}}\left(\tau_{i}\right)$, we can bound the second term as:

$$
\begin{aligned}
\left\|\nabla \cdot\left(\Pi_{h}^{p} \overrightarrow{\mathbf{H}}-\overrightarrow{\mathbf{H}}_{h}\right)\right\|_{0, \tau_{i}} & \leq C \frac{p_{i}^{2}}{h_{i}}\left\|\Pi_{h}^{p} \overrightarrow{\mathbf{H}}-\overrightarrow{\mathbf{H}}_{h}\right\|_{0, \tau_{i}} \\
& \leq C T \frac{p_{i}^{2}}{h_{i}} \frac{h_{i}^{\nu-1}}{p_{i}^{s-\frac{3}{2}}}\|(\overrightarrow{\mathbf{E}}, \overrightarrow{\mathbf{H}})\|_{s, \tau_{i}} \\
& \leq C T \frac{h_{i}^{\nu-2}}{p_{i}^{s-\frac{7}{2}}}\|(\overrightarrow{\mathbf{E}}, \overrightarrow{\mathbf{H}})\|_{s, \tau_{i}},
\end{aligned}
$$

by combining Eq. (3.19) with Eq. (3.22). An equivalent bound can be obtained for the divergence of $\overrightarrow{\mathbf{E}}_{h}$ in the case of a source free medium which, combined with the above, yields the result.

As could be expected, the result inherits the temporal linear growth from the convergence result and confirms the possibility of recovering spectral convergence of the divergence under the assumption of sufficient smoothness of the solutions. It should be noted that while the result confirms high-order accuracy and convergence, the estimate for the actual convergence rate is certainly suboptimal and leaves room for improvement. 


\section{Numerical experiments}

In the following, we shall discuss the validity of the main theoretical results of the previous sections through the numerical solution of the two-dimensional Maxwell equations in the TM polarization, i.e. we solve for $\left(H_{x}, H_{y}, E_{z}\right)$. To limit the scope of the presentation, we will focus our attention on the $\mathrm{LF}_{2}$ and $\mathrm{LF}_{4}$ schemes, since the $\mathrm{LF}_{4}$ scheme is preferable to any other higher order LF scheme as stated in Tab. 1. We denote by $\mathrm{CFL}\left(\mathrm{LF}_{N}\right)=\max _{i}\left(c_{i} \Delta t / h_{i}\right)$ the CFL number of the $\mathrm{LF}_{N}$ scheme, where $c_{i}$ is the local speed of propagation. In Tab. 2, we summarize the CFL values of the $\mathrm{LF}_{2}$ based DGTD- $\mathbb{P}_{p}$ method, where $p_{i}=p, \forall \tau_{i} \in \Omega_{h}$. If $p_{i}$ varies from element to element in the mesh, the DGTD- $\mathbb{P}_{p_{i}}$ method has the same stability limit as the DGTD- $\mathbb{P}_{\min \left\{p_{i}\right\}}$ method, as long as the mesh is actually refined. For instance, if $p=\left\{p_{1}, p_{2}, p_{3}\right\}=\{4,3,1\}$ then $\mathrm{CFL}\left(\mathrm{LF}_{2}, \mathrm{DGTD}-\mathbb{P}_{(4,3,1)}\right)=\mathrm{CFL}\left(\mathrm{LF}_{2}, \mathrm{DGTD}-\mathbb{P}_{1}\right)=0.3$. The CFL values of the $\mathrm{LF}_{4}$ schemes are given by $\mathrm{CFL}\left(\mathrm{LF}_{4}\right)=2.847 \mathrm{CFL}\left(\mathrm{LF}_{2}\right)$ (see Tab. 1 ).

Table 2: The CFL values of the $\mathrm{LF}_{2}$ based DGTD- $\mathbb{P}_{p}$ method.

\begin{tabular}{|c|c|c|c|c|c|c|c|c|c|c|}
\hline$p$ & $\mathbf{0}$ & $\mathbf{1}$ & $\mathbf{2}$ & $\mathbf{3}$ & $\mathbf{4}$ & $\mathbf{5}$ & $\mathbf{6}$ & $\mathbf{7}$ & $\mathbf{8}$ & $\mathbf{9}$ \\
\hline \hline $\mathrm{CFL}\left(\mathrm{LF}_{2}\right)$ & 1.0 & 0.3 & 0.2 & 0.1 & 0.08 & 0.06 & 0.045 & 0.035 & 0.03 & 0.025 \\
\hline
\end{tabular}

\subsection{Problem 1: Eigenmode in a PEC square cavity}

We consider the propagation of an eigenmode which is a standing wave of frequency $f=212 \mathrm{MHz}$ and wavelength $\lambda=1.4 \mathrm{~m}$ in a unitary metallic cavity with $\epsilon=\mu=$ 1 in normalized units. Owing to the existence of an exact analytical solution, this problem allows us to appreciate the numerical results at any point and time in the cavity. Numerical simulations make use of a non-conforming locally refined triangular meshes of the square $[0,1] \times[0,1]$ as shown on Fig. 1 . For a given non-conforming mesh, we assign to coarse (i.e. non refined) elements a high polynomial degree $p_{1}$ and to refined region a low polynomial degree $p_{2}$ (see [8]). The resulting method is referred to as DGTD- $\mathbb{P}_{\left(p_{1}, p_{2}\right)}$. If $p_{1}=p_{2}=p$, the scheme is simply called DGTD- $\mathbb{P}_{p}$. In the sequel, we compare the $\mathrm{LF}_{2}$ and $\mathrm{LF}_{4}$ schemes using the DGTD- $\mathbb{P}_{p}$ and DGTD-P $\left.\mathbb{( p}_{1}, p_{2}\right)$ methods.

As a first verification of the theoretical estimates, we consider a non-conforming mesh consists of 152 triangles (128 of them in the refined region) and 97 nodes (24 of them are hanging nodes). All simulations are carried out for time $T=90$ which corresponds to 64 periods. We plot on Fig. 2 the time evolution of the $L^{2}$ error of the DGTD- $\mathbb{P}_{p}$ and DGTD-P $\mathbb{P}_{\left(p_{1}, p_{2}\right)}$ methods using the $\mathrm{LF}_{2}$ and $\mathrm{LF}_{4}$ schemes. It can be seen from Fig. 2 that the gain in the $L^{2}$ error is notable when the accuracy in space and time are increased. Tab. 3 gives the final $L^{2}$ error, the number of degrees of freedom (\# DOF) and the CPU time in seconds to reach time $T=90$. From Tab. 3 we observe that the $\mathrm{LF}_{4}$ scheme requires almost 1.5 times less CPU time and it is at least 4 times (for $p, p_{1}=3$ ), 20 times (for $p, p_{1}=4$ ) and 120 times (for $p, p_{1}=5$ ) more accurate than the $\mathrm{LF}_{2}$ scheme based on the observed $L^{2}$ errors. Furthermore, for a given accuracy, the $\mathrm{LF}_{4}$ based DGTD- 


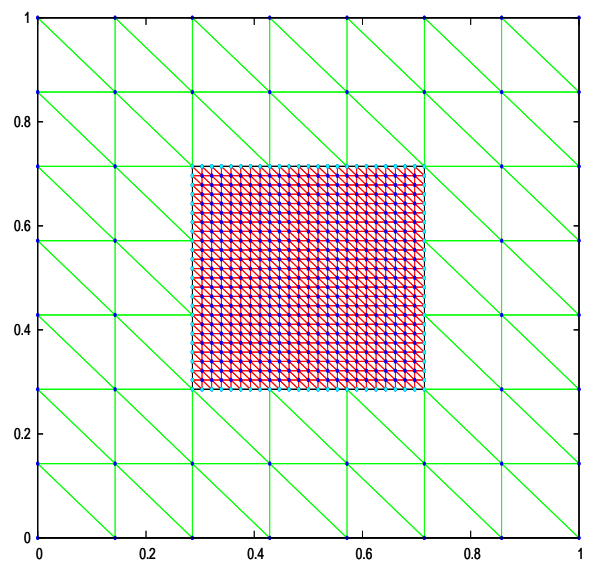

Figure 1: Problem 1: Example of a non-conforming locally refined triangular mesh.

$\mathbb{P}_{\left(p_{1}, p_{2}\right)}$ method requires less CPU time and less degrees of freedom than the $\mathrm{LF}_{4}$ based DGTD-P $\mathbb{P}_{p}$ method.

Fig. 3 illustrates the numerical $h$-convergence of the DGTD- $\mathbb{P}_{p}$ and DGTD- $\mathbb{P}_{\left(p_{1}, p_{2}\right)}$ methods. Corresponding asymptotic convergence orders are summarized in Tab. 4. As it could be expected from the use of a $N$ th accurate time integration scheme, the asymptotic convergence order is bounded by $N$ independently of the approximation order $p$. On Fig. 4 we show the numerical $p$-convergence of the DGTD- $\mathbb{P}_{p}$ and DGTD$\mathbb{P}_{\left(p_{1}, p_{2}\right)}$ methods for different approximation orders $p$ and different mesh resolutions $h$. Following the main result, Theorem 3.1, we expect that the error grows at most linearly in time and that the growth rate should vanish spectrally for smooth solution. The results on Fig. 4 not only confirm the validity of both statements but also illustrate that Theorem 3.1 is sharp, i.e. we cannot in general guarantee slower than linear growth, although we can control the growth rate by the approximation order $p$.

Table 3: Problem 1: $L^{2}$-error, CPU time in seconds and \# DOF to reach time $T=90$ using the $\mathrm{LF}_{2}$ and $\mathrm{LF}_{4}$ based DGTD methods.

\begin{tabular}{|c|c||cc||cc|}
\hline \multicolumn{2}{|c||}{ DGTD- $\mathbb{P}_{p}$ method } & \multicolumn{2}{c||}{$\mathrm{LF}_{2}$ scheme } & \multicolumn{2}{c|}{ LF $_{4}$ scheme } \\
\hline$p$ & \# DOF & Error & CPU time & Error & CPU time \\
\hline $\mathbf{2}$ & 912 & $4.9 \mathrm{E}-02$ & $25 \mathrm{~s}$ & $3.6 \mathrm{E}-02$ & $17 \mathrm{~s}$ \\
$\mathbf{3}$ & 1520 & $3.6 \mathrm{E}-03$ & $76 \mathrm{~s}$ & $8.5 \mathrm{E}-04$ & $54 \mathrm{~s}$ \\
$\mathbf{4}$ & 2280 & $2.0 \mathrm{E}-03$ & $161 \mathrm{~s}$ & $9.2 \mathrm{E}-05$ & $110 \mathrm{~s}$ \\
$\mathbf{5}$ & 3192 & $1.1 \mathrm{E}-03$ & $364 \mathrm{~s}$ & $9.3 \mathrm{E}-06$ & $251 \mathrm{~s}$ \\
\hline
\end{tabular}

\begin{tabular}{|c|c||cc||cc|}
\hline \multicolumn{2}{|c||}{ DGTD- $\mathbb{P}_{\left(p_{1}, p_{2}\right)}$ method } & \multicolumn{2}{c||}{ LF $_{2}$ scheme } & \multicolumn{2}{c|}{ LF $_{4}$ scheme } \\
\hline$\left(p_{1}, p_{2}\right)$ & \# DOF & Error & CPU time & Error & CPU time \\
\hline $\mathbf{( 3 , 2 )}$ & 1008 & $1.3 \mathrm{E}-02$ & $29 \mathrm{~s}$ & $8.6 \mathrm{E}-04$ & $20 \mathrm{~s}$ \\
$\mathbf{( 4 , 3 )}$ & 1640 & $3.2 \mathrm{E}-03$ & $86 \mathrm{~s}$ & $9.6 \mathrm{E}-05$ & $60 \mathrm{~s}$ \\
$\mathbf{( 5 , 4 )}$ & 2424 & $2.0 \mathrm{E}-03$ & $183 \mathrm{~s}$ & $9.4 \mathrm{E}-06$ & $125 \mathrm{~s}$ \\
\hline
\end{tabular}

We conclude this experimental study by considering the numerical behavior of the 

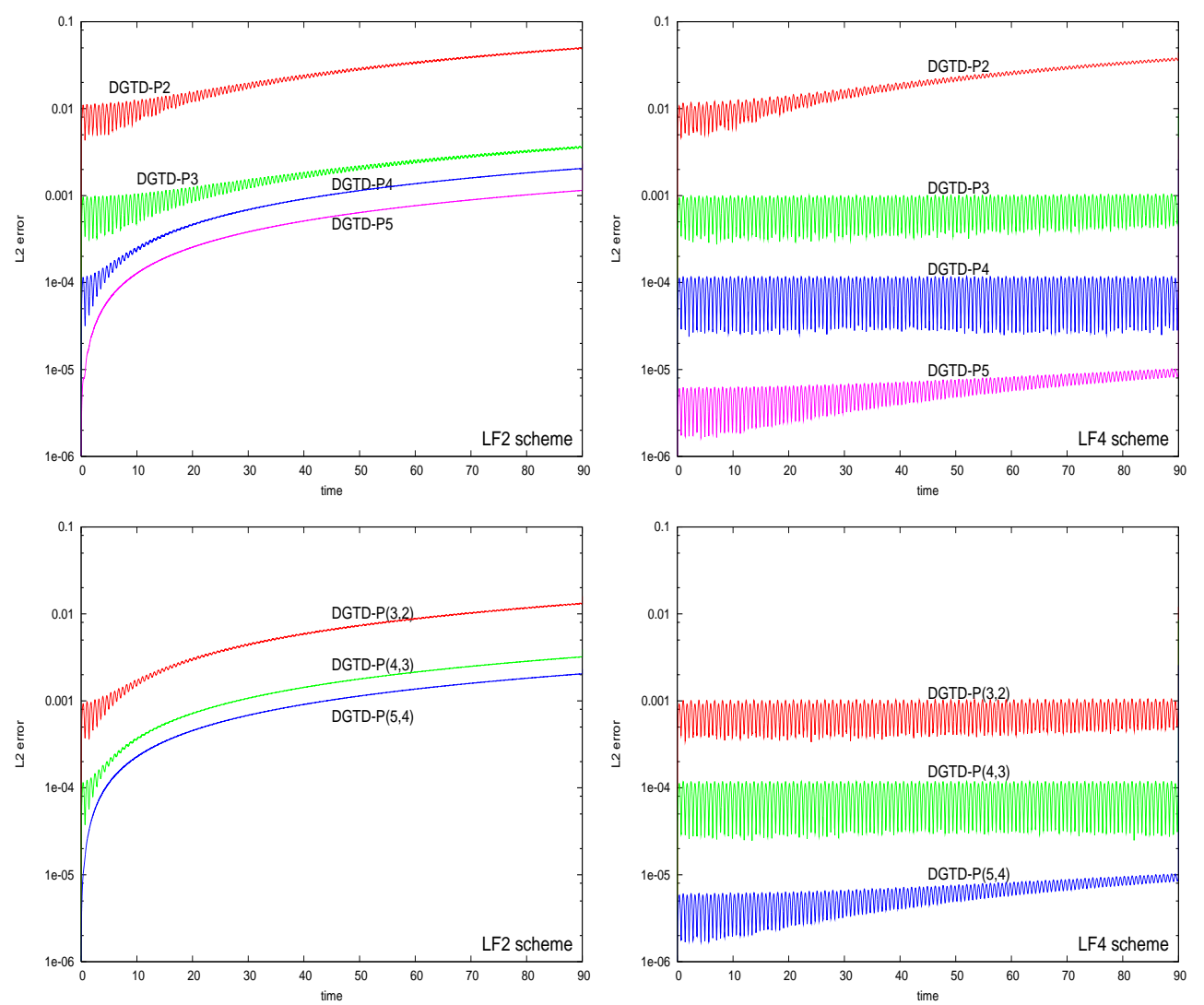

Figure 2: Problem 1: Time evolution of the $L^{2}$ error. DGTD- $\mathbb{P}_{p}$ (top) and DGTD- $\mathbb{P}_{\left(p_{1}, p_{2}\right)}$ (bottom) methods using the $\mathrm{LF}_{2}$ (left) and $\mathrm{LF}_{4}$ (right) schemes.

divergence error. For this purpose, we still consider the eigenmode problem. The computational domain is discretized by a non-conforming locally refined mesh with 48 triangles (32 of them in the refined region) and 37 nodes (16 of them are hanging nodes), which corresponds to a grid resolution of 5 points per wavelength. Simulations are carried out for time $T=30$ which corresponds to 20 periods. Fig. 5 shows the global $L^{2}$ error of the divergence of $\overrightarrow{\mathbf{H}}$ as a function of time and the approximation order $p$ using respectively the DGTD- $\mathbb{P}_{p}$ and DGTD- $\mathbb{P}_{\left(p_{1}, p_{2}\right)}$ methods. The results in Fig. 5 confirm that the method preserves the divergence error to the order of approximation, i.e., it decays spectrally (for $N=4$ ) with increasing polynomial order.

On Fig. 6 we show the numerical $h$ - and $p$-convergence of the divergence of $\overrightarrow{\mathbf{H}}$ using the $\mathrm{LF}_{2}$ and $\mathrm{LF}_{4}$ schemes. Consistent with the theoretical result in Theorem 3.2, the divergence error vanishes spectrally as we increase the approximation order $p$. Corresponding asymptotic convergence orders of the divergence of $\overrightarrow{\mathbf{H}}$ are given in Tab. 5 . One can observe that the convergence order is bounded by $N+2$ contrary to what we have observed for the $h$-convergence of the DGTD methods which confirms that the estimate given in Eq. (3.23) is suboptimal and leaves room for improvement. 

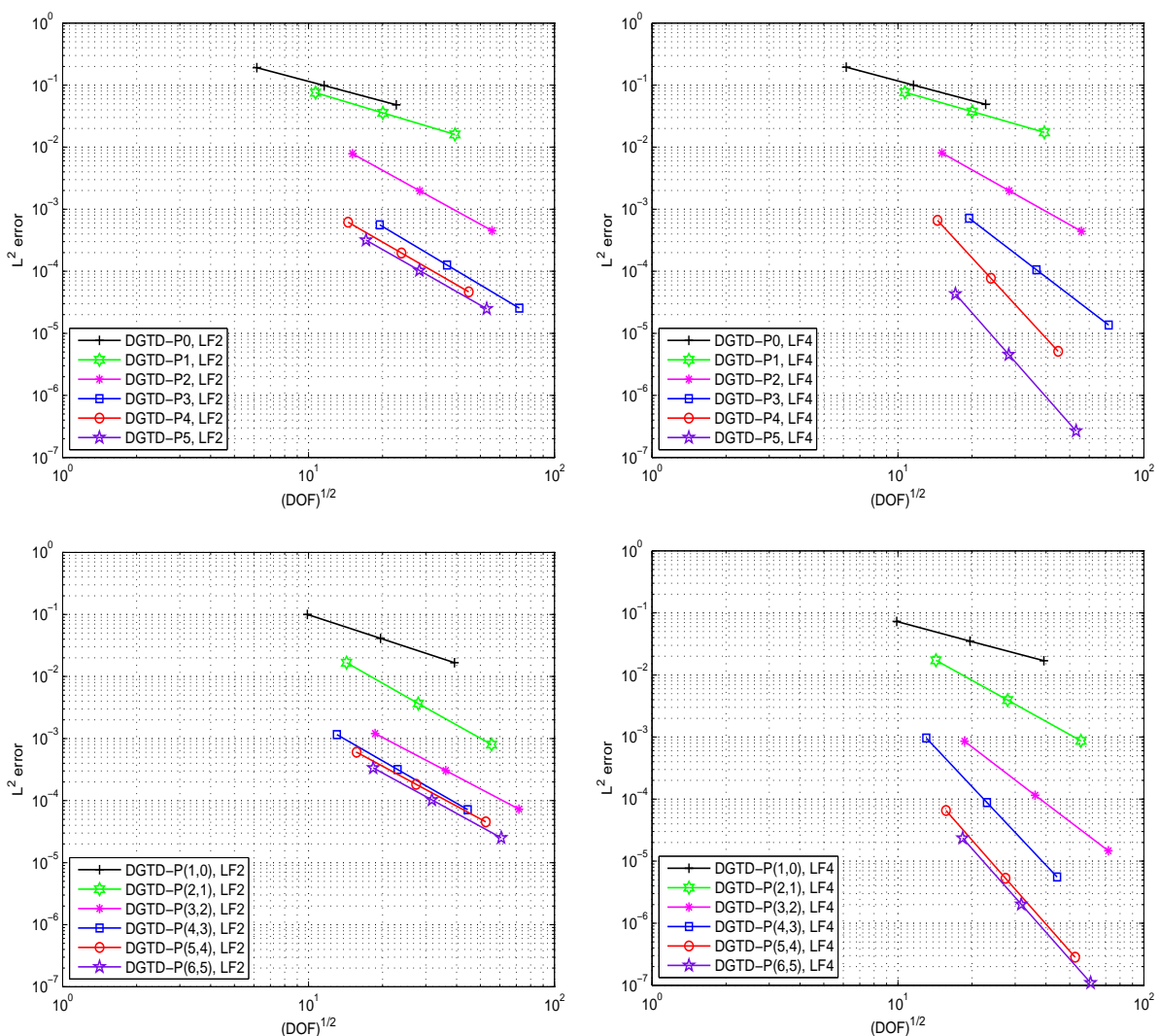

Figure 3: Problem 1: $h$-convergence of the DGTD- $\mathbb{P}_{p}$ (top) and DGTD- $\mathbb{P}_{\left(p_{1}, p_{2}\right)}$ (bottom) methods using the $\mathrm{LF}_{2}$ (left) and $\mathrm{LF}_{4}$ (right) schemes. $L^{2}$ error at time $T=2$ as a function of the square root of \#DOF.

\subsection{Problem 2: scattering by a multilayered dielectric circular cylinder}

Having verified the performance of the basic computational setup as well as the theoretical estimates, let us now consider a non-trivial problem of more realistic character. In this section, we shall only consider the $\mathrm{LF}_{4}$ scheme, and our objective is to compare the non-conforming DGTD method proposed in this paper and the conforming DGTD method studied in [10].

We consider a problem, in which a plane wave impinges on a dielectric cylinder with multiple layers, experiencing reflections and refractions at the material interfaces. The problem setting is shown on Fig. 7 . We assume that the cylinder is illuminated by a monochromatic plane wave of the form:

$$
E_{z}^{i n c}=\exp \left(-\mathrm{i}\left(k_{6} x-\omega t\right)\right), H_{y}^{i n c}=-\exp \left(-\mathrm{i}\left(k_{6} x-\omega t\right)\right),
$$

where $k_{6}=\omega \sqrt{\epsilon_{6} \mu_{6}}$. We suppose that the cylinder contains five layers which correspond to five concentric cylinders. The radii of the cylinders are $R_{1}=0.1, R_{2}=$ $0.2, R_{3}=0.3, R_{4}=0.4$ and $R_{5}=0.5$. Each layer consists of a dielectric non-magnetic 
Table 4: Problem 1: Asymptotic convergence orders of the $\mathrm{LF}_{2}$ and $\mathrm{LF}_{4}$ based DGTD methods.

\begin{tabular}{|c|cccccc|}
\hline DGTD- $\mathbb{P}_{p}$ method, $p=$ & $\mathbf{0}$ & $\mathbf{1}$ & $\mathbf{2}$ & $\mathbf{3}$ & $\mathbf{4}$ & $\mathbf{5}$ \\
\hline $\mathrm{LF}_{2}$ scheme & 1.06 & 1.19 & 2.18 & 2.37 & 2.29 & 2.25 \\
$\mathrm{LF}_{4}$ scheme & 1.06 & 1.14 & 2.23 & 3.03 & 4.30 & 4.50 \\
\hline DGTD-P ${ }_{\left(p_{1}, p_{2}\right)}$ method, $\left(p_{1}, p_{2}\right)=$ & $\mathbf{( 1 , 0 )}$ & $\mathbf{( 2 , 1 )}$ & $\mathbf{( 3 , 2 )}$ & $\mathbf{( 4 , 3 )}$ & $\mathbf{( 5 , 4 )}$ & $\mathbf{( 6 , 5 )}$ \\
\hline $\mathrm{LF}_{2}$ scheme & 1.30 & 2.23 & 2.08 & 2.27 & 2.13 & 2.17 \\
$\mathrm{LF}_{4}$ scheme & 1.05 & 2.20 & 3.01 & 4.21 & 4.50 & 4.48 \\
\hline
\end{tabular}

Table 5: Problem 1: Asymptotic convergence orders of the divergence of $\overrightarrow{\mathbf{H}}$.

\begin{tabular}{|c|cccccc|}
\hline DGTD- $\mathbb{P}_{p}$ method, $p=$ & $\mathbf{1}$ & $\mathbf{2}$ & $\mathbf{3}$ & $\mathbf{4}$ & $\mathbf{5}$ & $\mathbf{6}$ \\
\hline $\mathrm{LF}_{2}$ scheme & 0.89 & 2.10 & 2.94 & 4.07 & 3.49 & 3.45 \\
$\mathrm{LF}_{4}$ scheme & 0.97 & 2.05 & 3.00 & 4.09 & 4.58 & 5.66 \\
\hline DGTD- $\mathbb{P}_{\left(p_{1}, p_{2}\right)}$ method, $\left(p_{1}, p_{2}\right)=$ & $\mathbf{( 2 , 1 )}$ & $\mathbf{( 3 , 2 )}$ & $\mathbf{( 4 , 3 )}$ & $\mathbf{( 5 , 4 )}$ & $\mathbf{( 6 , 5 )}$ & \\
\hline $\mathrm{LF}_{2}$ scheme & 2.33 & 2.81 & 3.84 & 3.24 & 3.46 & \\
$\mathrm{LF}_{4}$ scheme & 2.26 & 2.73 & 3.94 & 4.40 & 5.50 & \\
\hline
\end{tabular}

material, i.e. $\mu_{i}=1, \epsilon_{i} \geq 1, i=1, \ldots, 6$. The characteristics of the materials and the corresponding wavelength in the different regions are given in Tab. 6. The angular frequency is $\omega=2 \pi$ and the wavelength in the vacuum is $\lambda=1$.

Table 6: Problem 2: Characteristics of the material in the different regions.

\begin{tabular}{|c|c|c|c|c|c|c|}
\hline Region & Region 1 & Region 2 & Region 3 & Region 4 & Region 5 & Region 6 \\
& $r<R_{1}$ & $R_{1}<r<R_{2}$ & $R_{2}<r<R_{3}$ & $R_{3}<r<R_{4}$ & $R_{4}<r<R_{5}$ & $r>R_{5}$ \\
\hline$\epsilon_{r}$ & $\epsilon_{1}=1$ & $\epsilon_{2}=4$ & $\epsilon_{3}=9$ & $\epsilon_{4}=16$ & $\epsilon_{5}=64$ & $\epsilon_{6}=1$ \\
$\lambda(\mathrm{m})$ & 1 & 0.5 & 0.33 & 0.25 & 0.125 & 1 \\
\hline
\end{tabular}

The computational domain is chosen as a cylinder of radius $R_{6}=1$, and is truncated with a first-order Silver-Müller absorbing boundary condition $\vec{n} \times \mathbf{E}=-c \mu \vec{n} \times(\vec{n} \times \mathbf{\mathbf { H }})$, where $c=1 / \sqrt{\epsilon \mu}$ is the speed of propagation. In this special case, no exact analytical solution is available for this problem; instead, we replace it by a reference solution obtained using the $\mathrm{LF}_{4}$ based DGTD- $\mathbb{P}_{6}$ method applied to a high resolution conforming mesh consisting of 25001 nodes and 49750 triangles. Contour lines of the $E_{z}$ and $H_{y}$ components at time $T=5$ are shown on Fig. 8 .

To show the effectiveness of the proposed method, we aim at making a comparison between the conforming DGTD method studied in [10] and the non-conforming DGTD method considered here. To this end, we first construct a conforming mesh consisting of 14401 nodes and 28560 triangles and we use different DGTD- $\mathbb{P}_{p}$ method, where the interpolation degree $p$ is uniform in space. Then, a non-conforming mesh is obtained by locally refining a coarse conforming mesh with a resolution of 10 points per the larger wavelength. The level of refinement depends on the local wavelength in each region. For example, the fifth region is refined four times since it corresponds to the lower wavelength. For this non-conforming mesh, we assign to each region a polynomial 

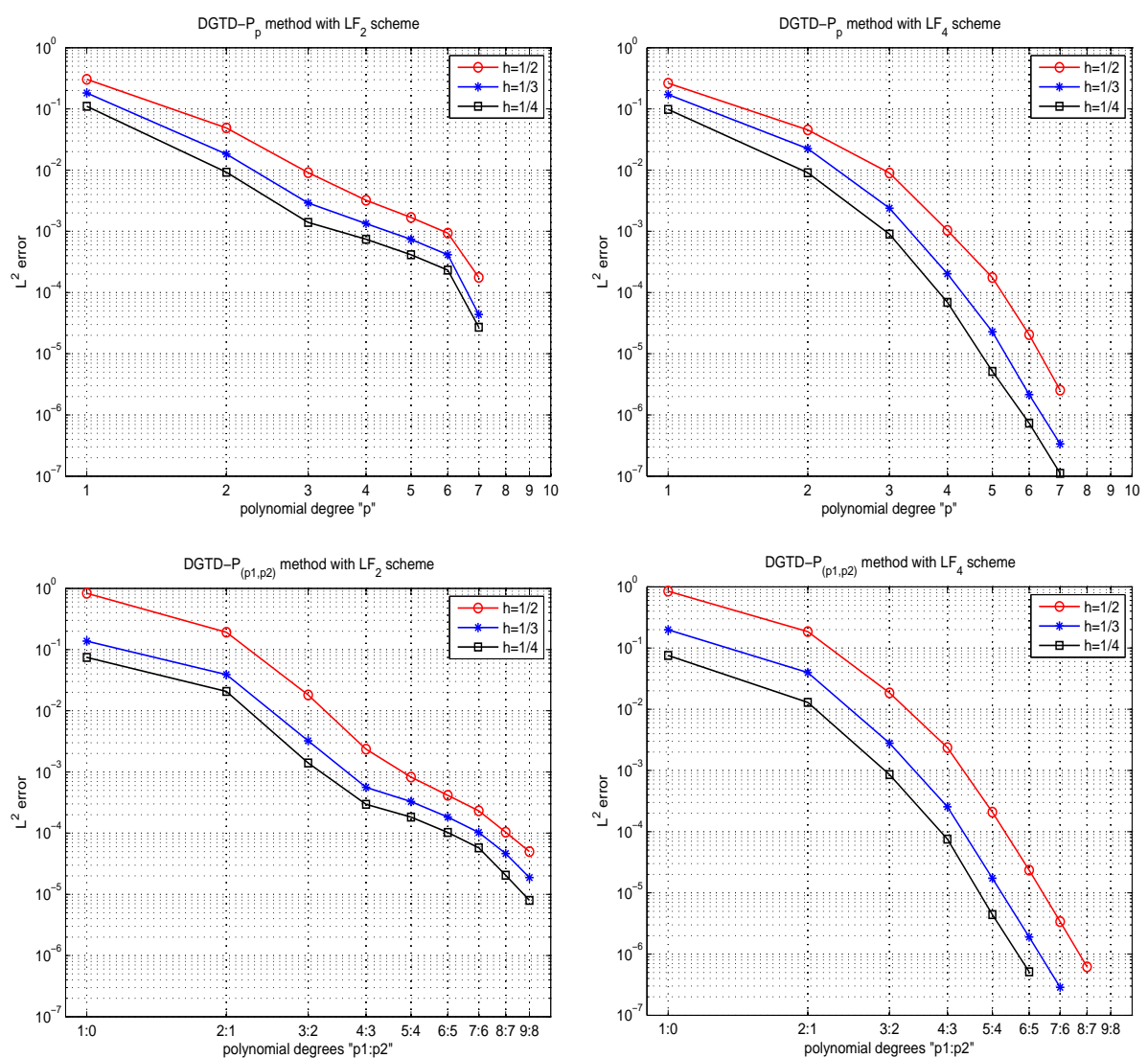

Figure 4: Problem 1: $p$-convergence of the DGTD- $\mathbb{P}_{p}$ (top) and DGTD- $\mathbb{P}_{\left(p_{1}, p_{2}\right)}$ (bottom) methods using the $\mathrm{LF}_{2}$ (left) and $\mathrm{LF}_{4}$ (right) schemes. $L^{2}$ error at time $T=2$ as a function of the approximation order $p$.

degree $p_{i}$ and we use different DGTD- $\mathbb{P}_{p_{i}}$ methods. The resulting non-conforming mesh consists of 27640 triangles and 14441 nodes in which 920 are hanging nodes (see Fig. 9). The level of refinement and the distribution of triangles in each region are summarized in Tab. 7.

Results are shown on Fig. 10 in terms of the $x$-wise 1D distribution along $y=0.0 \mathrm{~m}$ of the $E_{z}$ and $H_{y}$ components. One can observe that the $H_{y}$ component is of low regularity and the proposed non-conforming DGTD- $\mathbb{P}_{p_{i}}$ method treats very well the discontinuity at the material interfaces. Although, the levels of refinement in regions 4 and 5 as well as the size of the jump in $\epsilon$ on the materials interfaces are high, and the mesh in regions $1,2,3,6$ are characterized by a few points per wavelength. We give in Tab. 8 the $L^{2}$ error with the reference solution, the CPU time and \# DOF to reach time $T=5$, for some cases of the conforming and non-conforming DGTD methods. As expected, the gain in CPU time between the two methods is notable. For instance, the DGTD- $\mathbb{P}_{(4,3,2,1,0,2)}$ method is roughly 2.3 times (for $H_{y}$ ) and 6.5 times (for $E_{z}$ ) more accurate and requires 11 times less $\mathrm{CPU}$ time and 1.7 times less memory than the conforming DGTD- $\mathbb{P}_{1}$ method. Moreover, the DGTD- $\mathbb{P}_{(4,3,2,2,1,4)}$ method requires 

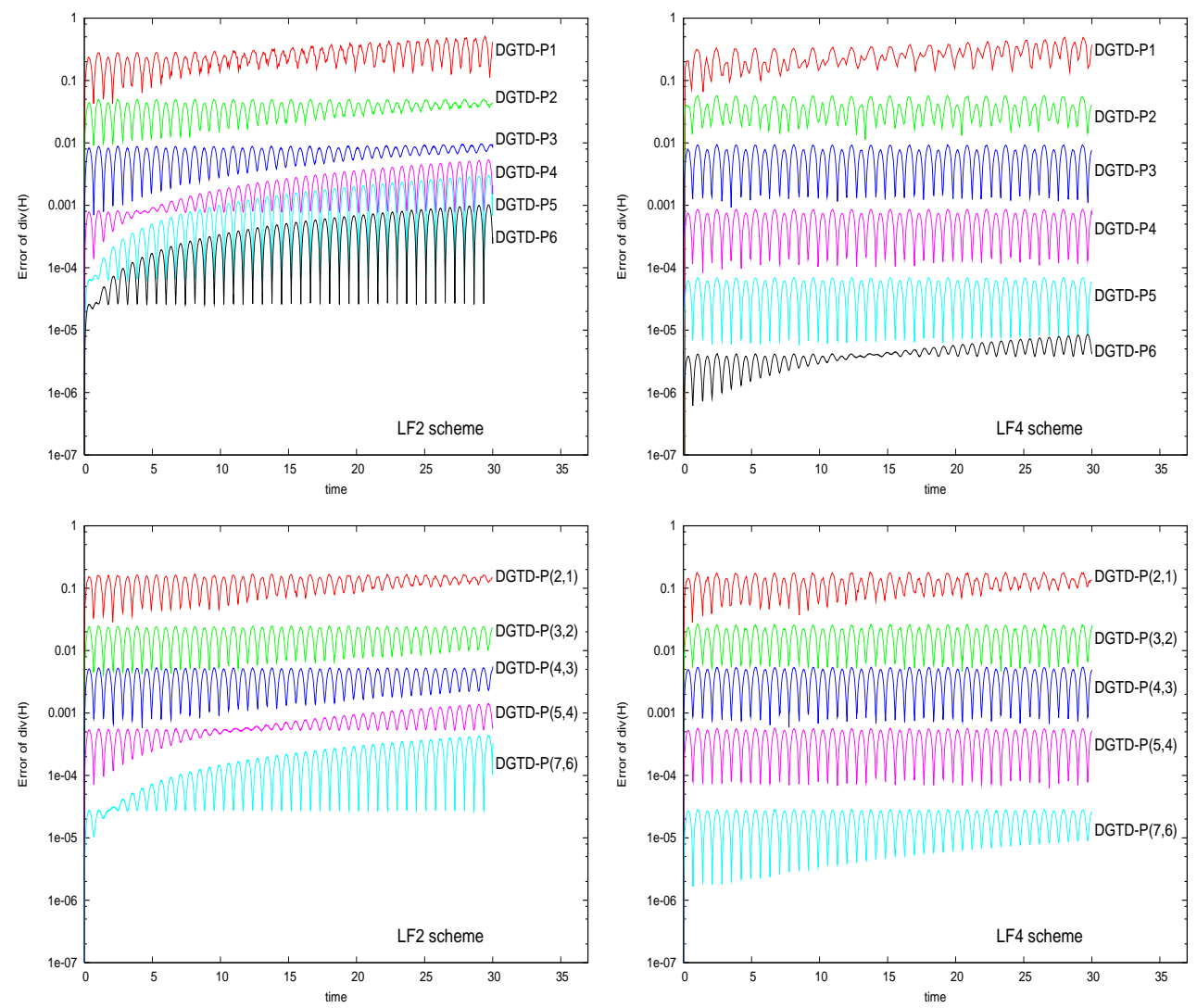

Figure 5: Problem 1: Global $L^{2}$ error of the divergence of $\overrightarrow{\mathbf{H}}$ as a function of time and $p$. DGTD- $\mathbb{P}_{p}$ (top) and DGTD- $\mathbb{P}_{\left(p_{1}, p_{2}\right)}$ (bottom) methods using the $\mathrm{LF}_{2}$ (left) and $\mathrm{LF}_{4}$ (right) schemes.

respectively 17 times and 50 times less CPU time than the conforming DGTD- $\mathbb{P}_{2}$ and DGTD- $\mathbb{P}_{3}$ methods.

\section{Concluding remarks}

The main purpose of this paper has been to study both theoretically and numerically an arbitrarily high-order DGTD method for the discretization of the time-domain Maxwell equations on non-conforming simplicial meshes. The central element which distinguishes the current work from previous attempts to develop such DGTD methods is that a high-order leap-frog time integration scheme is adopted here instead of a high-order Runge-Kutta method. We have proved that the resulting DGTD method is stable under some CFL-type condition. Also, we have developed a complete, if not optimal, convergence theory. We have confirmed the results of the analysis by thorough numerical experiments in two space dimensions, illustrating the flexibility, versatility, and efficiency of the proposed arbitrarily high-order DGTD method. 

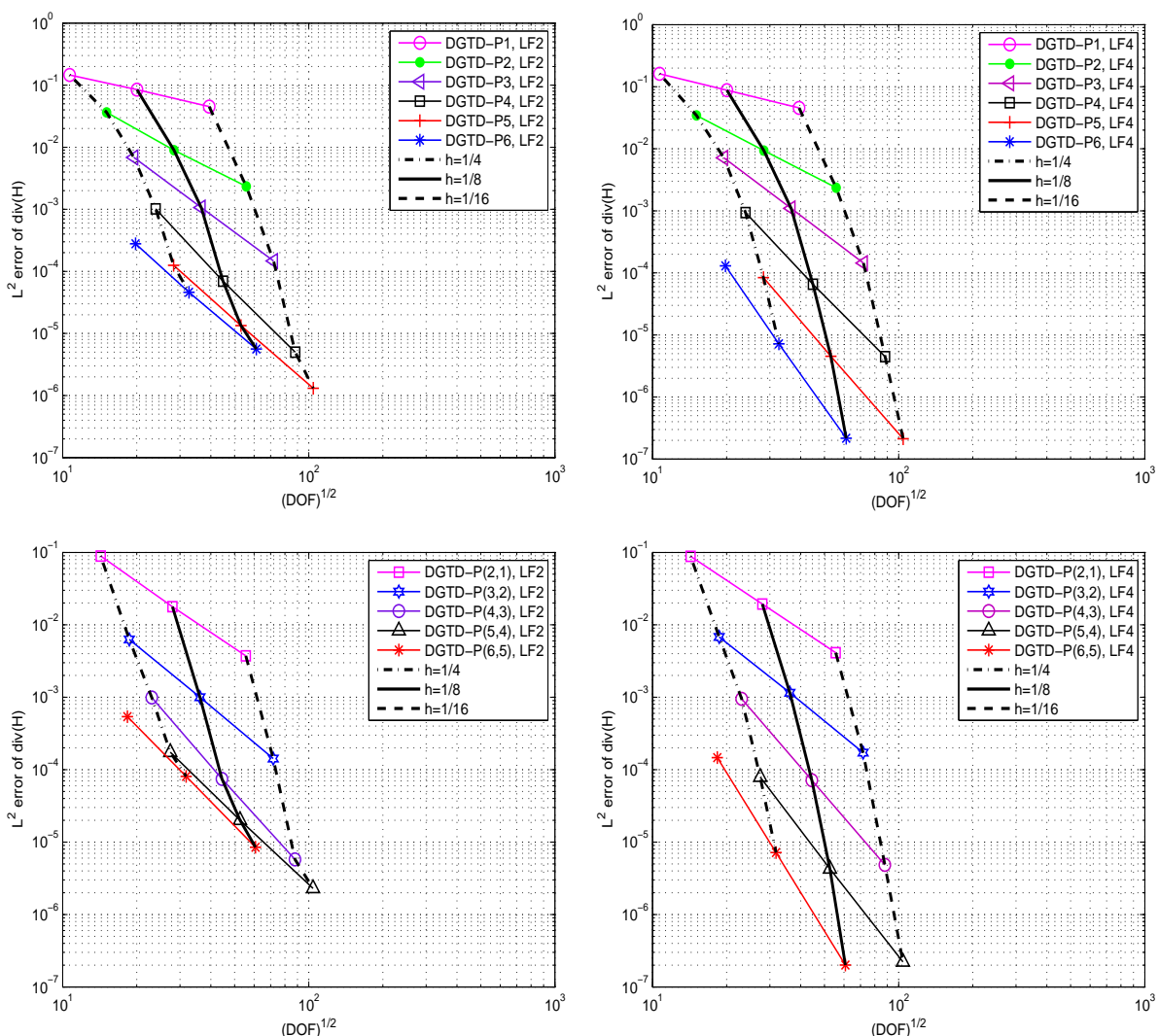

Figure 6: Problem 1: $h$ - and $p$-convergence of the divergence of $\overrightarrow{\mathbf{H}}$. DGTD- $\mathbb{P}_{p}$ (top) and DGTD$\mathbb{P}_{\left(p_{1}, p_{2}\right)}$ (bottom) methods using the $\mathrm{LF}_{2}$ (left) and $\mathrm{LF}_{4}$ (right) schemes. Errors evaluated at time $T=2$.

\section{Acknowledgment}

This research was partially supported by a grant from the French National Ministry of Education and Research (MENSR, 19755-2005). The author wish to express his gratitude to Stéphane Lanteri and Ronan Perrussel for helpful comments. The author also extends his appreciation to the referees for their helpful suggestions.

\section{References}

[1] M. Bernacki, L. Fezoui, S. LANTERI, AND S. Piperno, Parallel unstructured mesh solvers for heterogeneous wave propagation problems, Appl. Math. Model., 30 (2006), pp. 744 763.

[2] I. BABUSKA AND M. SURI, The hp-version of the finite element method with quasiuniform meshes, RAIRO: Math. Model. Numer. Anal., 21 (1987), pp. 199-238.

[3] M.-H. CHEN, B. COCKBURN, AND F. REITICH, High-order RKDG methods for computational electromagnetics, J. Sci. Comput., 22 (2005), pp. 205-226. 


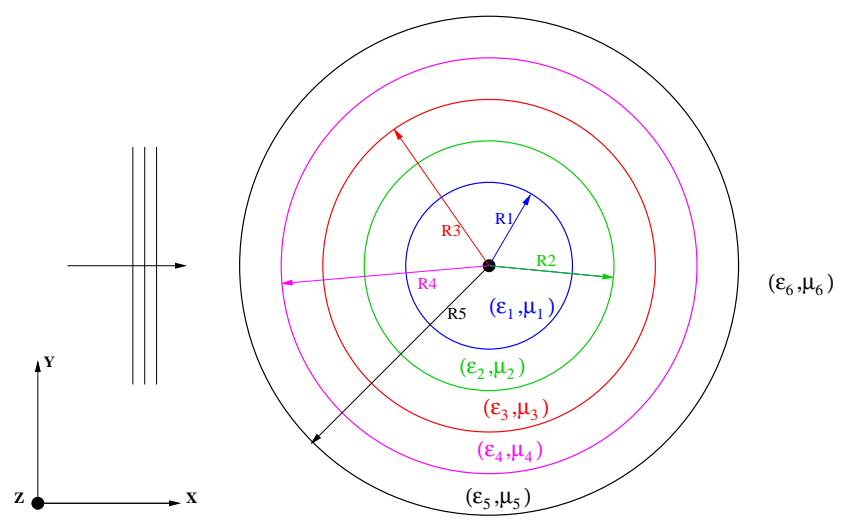

Figure 7: Problem 2: Computational domain and problem setting.
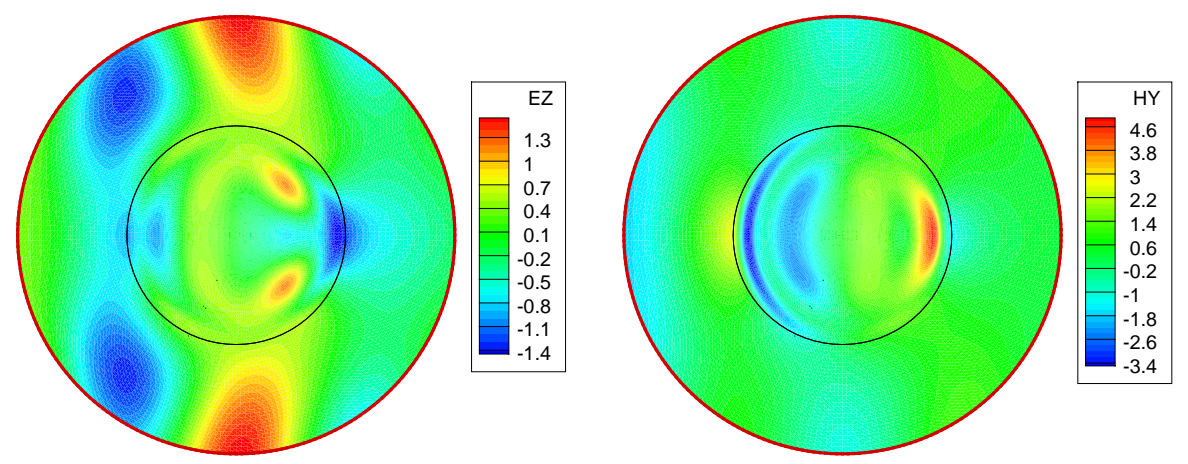

Figure 8: Problem 2: Contour lines of the reference solution at time $T=5$.

[4] E. J. ChUng AND B. ENGQUist, Optimal discontinuous Galerkin methods for wave propagation, SIAM J. Numer. Anal., 44 (2006), pp. 2131-2158.

[5] G. Cohen, X. Ferrieres, AND S. Pernet, A spatial high-order hexahedral discontinuous Galerkin method to solve Maxwell's equations in time domain, J. Comput. Phys., 217 (2006), pp. 340-363.

[6] M. H. CARPENTER AND C. A. Kennedy, Fourth-order 2N-storage Runge-Kutta schemes, NASA-TM-109112, NASA Langley Research center, VA, 1994.

[7] R. Cools, An encyclopedia of cubature formulas, J. Complexity, 19 (2003), pp. 445-453.

[8] H. FAHS, Development of a hp-like discontinuous Galerkin time-domain method on non-

Table 7: Problem 2: \# triangles and the level of refinement in each region.

\begin{tabular}{|c|c|c|c|c|c|c|}
\hline Region & Reg. 1 & Reg. 2 & Reg. 3 & Reg. 4 & Reg. 5 & Reg. 6 \\
\hline Interpolation order & $p_{1}$ & $p_{2}$ & $p_{3}$ & $p_{4}$ & $p_{5}$ & $p_{6}$ \\
$\begin{array}{c}\text { Level of refinement } \\
\text { \# triangles } \\
\text { non-conforming mesh } \\
\text { \# triangles } \\
\text { conforming mesh }\end{array}$ & 40 & 1 & 2 & 3 & 4 & 0 \\
& 2640 & 2880 & 1280 & 5120 & 20480 & 400 \\
\hline
\end{tabular}



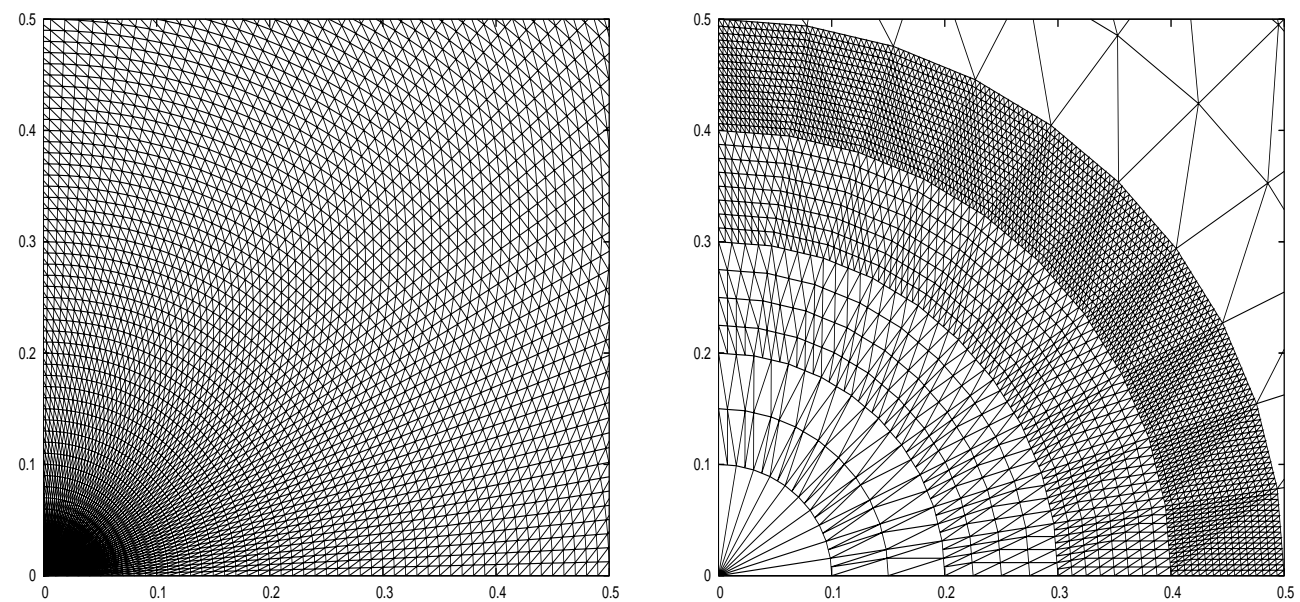

Figure 9: Problem 2: Conforming mesh (left) and non-conforming mesh (right).

Table 8: Problem 2: Relative errors, CPU time in minutes and \# DOF to reach time $T=5$.

\begin{tabular}{|c|c|c|c|c|}
\hline \multicolumn{5}{|c|}{ LF $_{4}$ based DGTD- $\mathbb{P}_{p}$ method using the conforming mesh } \\
\hline DGTD- $\mathbb{P}_{p}$ & Error on $H_{y}$ & Error on $E_{z}$ & CPU time & \# DOF \\
\hline DGTD- $\mathbb{P}_{0}$ & $8.6 \%$ & $12.7 \%$ & $25 \mathrm{~min}$ & 28560 \\
DGTD- $\mathbb{P}_{1}$ & $7.6 \%$ & $7.80 \%$ & $137 \mathrm{~min}$ & 85680 \\
DGTD- $\mathbb{P}_{2}$ & $2.2 \%$ & $1.20 \%$ & $286 \mathrm{~min}$ & 171360 \\
DGTD- $\mathbb{P}_{3}$ & $1.6 \%$ & $0.90 \%$ & $842 \mathrm{~min}$ & 285600 \\
\hline
\end{tabular}

\begin{tabular}{|c|c|c|c|c|}
\hline \multicolumn{5}{|c|}{ LF $_{4}$ based DGTD- $\mathbb{P}_{p_{i}}$ method using the non-conforming mesh } \\
\hline DGTD- $\mathbb{P}_{\left(p_{1}, p_{2}, p_{3}, p_{4}, p_{5}, p_{6}\right)}$ & Error on $H_{y}$ & Error on $E_{z}$ & CPU time & \# DOF \\
\hline DGTD-P $\mathbb{P}_{(4,3,2,1,0,2)}$ & $3.3 \%$ & $1.2 \%$ & $12.0 \mathrm{~min}$ & 49720 \\
\hline DGTD-P $\mathbb{P}_{(4,3,2,2,0,2)}$ & $2.8 \%$ & $1.2 \%$ & $12.5 \mathrm{~min}$ & 65080 \\
\hline DGTD-P $\mathbb{P}_{(4,3,2,2,1,4)}$ & $1.7 \%$ & $0.9 \%$ & $17.0 \mathrm{~min}$ & 109640 \\
\hline DGTD-P $\mathbb{P}_{(4,2,2,4,1,4)}$ & $1.4 \%$ & $0.8 \%$ & $21.0 \mathrm{~min}$ & 154440 \\
\hline
\end{tabular}

conforming simplicial meshes for electromagnetic wave propagation, Int. J. Numer. Anal. Model., 6 (2009), pp. 193-216.

[9] H. FAHS, L. FEzOUI, S. LANTERI, AND F. RAPETTI, Preliminary investigation of a nonconforming discontinuous Galerkin method for solving the time domain Maxwell equations, IEEE Trans. on Magnet., 44 (2008), pp. 1254-1257.

[10] L. Fezoui, S. LANTERI, S. LOHREnGEL, AND S. Piperno, Convergence and stability of a discontinuous Galerkin time-domain method for the heterogeneous Maxwell equations on unstructured meshes, ESAIM: Math. Model. Numer. Anal., 39 (2005), pp. 1149-1176.

[11] J. M. Hyman And M. Shashkov, Mimetic Finite Difference Methods for Maxwell's Equations and the Equations of Magnetic Diffusion, J. Electromagn. Waves Appl., 15 (2001), pp. 107-108

[12] Bo HE AND F. L. TEIXEIRA, Sparse and explicit FETD via approximate inverse Hodge (mass) matrix, IEEE Microwave and Wireless Components Letters, 16 (2006), pp. 348-350.

[13] J. S. HESTHAVEN AND T. WARBURTON, Nodal high-order methods on unstructured grids. I. 

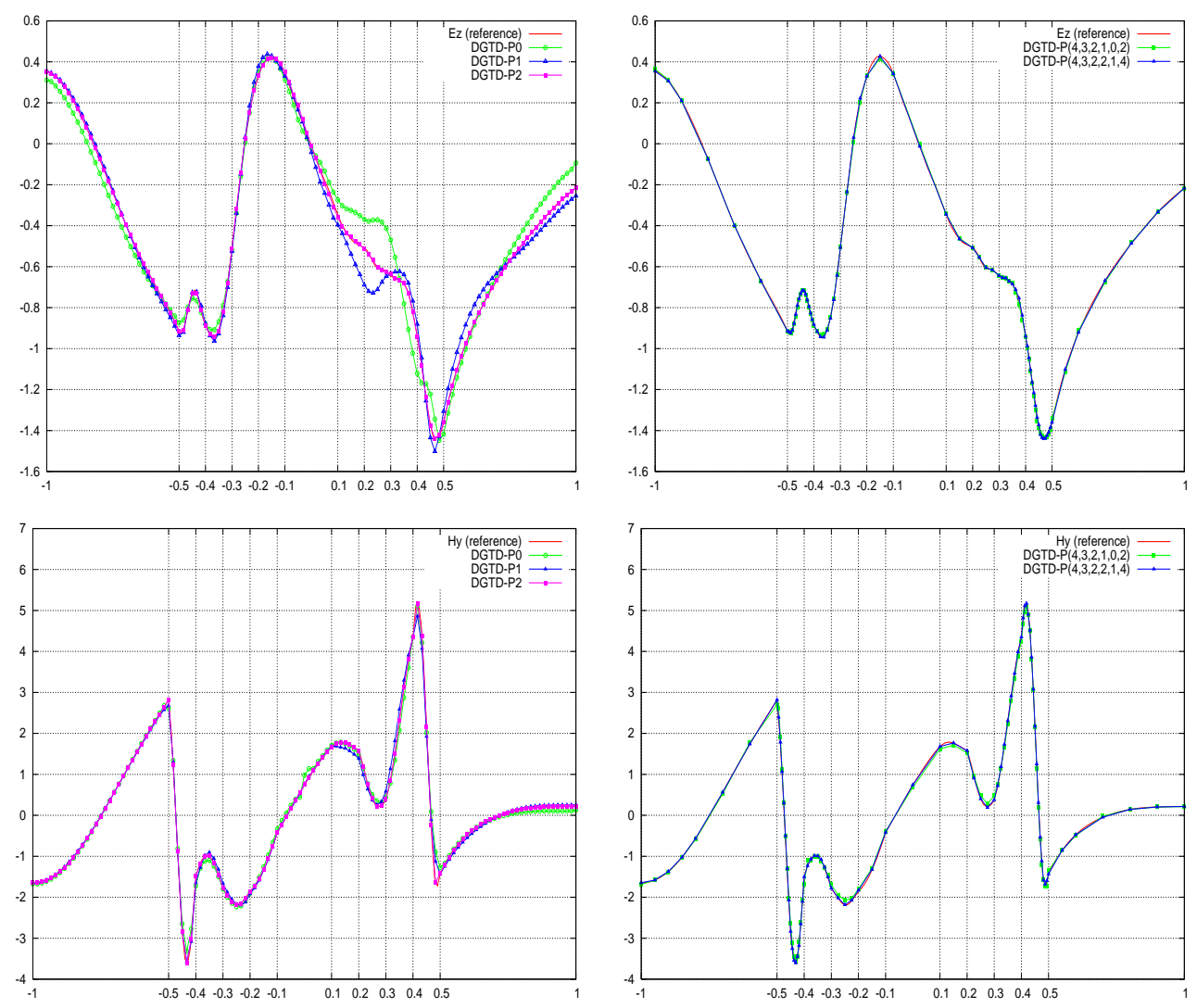

Figure 10: Problem 2: 1D distribution of the $E_{z}$ (top) and $H_{y}$ (bottom) components along $y=0.0$ at time $T=5$. Conforming DGTD- $\mathbb{P}_{p}$ method (left) and non-conforming DGTD- $\mathbb{P}_{p_{i}}$ method (right).

Time-domain solution of Maxwell's equations, J. Comput. Phys., 181 (2002), pp. 186-221.

[14] S. Jund AND S. SALmon, Arbitrary high-order finite element schemes and high-order mass lumping, Int. J. Appl. Math. Comput. Sci., 17 (2007), pp. 375-393.

[15] T. Lu, P. Zhang, AND W. CAI, Discontinuous Galerkin methods for dispersive and lossy Maxwell's equations and PML boundary conditions, J. Comput. Phys., 200 (2004), pp. 549-580.

[16] P. MonK AND J. RichteR, A discontinuous Galerkin method for linear symmetric hyperbolic systems in inhomogeneous media, SIAM J. Sci. Comput., 22 (2005), pp. 433-477.

[17] M. Remaki, Méthodes numéiques pour les équations de Maxwell instationnaires en milieu hétérogène, Ph.D. thesis, Ecole Nationale des Ponts et Chaussées, 1999.

[18] D. SÁRMÁNY, M. A. BotCheV, AND J.J.W. VAN DER VEGT, Dispersion and dissipation error in high-order Runge-Kutta discontinuous Galerkin discretisations of the Maxwell equations, J. Sci. Comput., 33 (2007), pp. 47-74.

[19] CH. SCHWAB, $p$ - and $h p$-finite element methods. Theory and applications to solid and fluid mechanics, Oxford University Press, Oxford, UK, 1998.

[20] K. S. YEE, Numerical solution of initial boundary value problems involving Maxwell's equations in isotropic media, IEEE Trans. Antennas and Propagat., 14 (1966), pp. 302-307.

[21] J. L. YounG, High-order, leapfrog methodology for the temporally dependent Maxwell's equations, Radio Sci., 36 (2001), pp. 9-17. 